ARTICLE

\title{
Inhibition of PDE4B suppresses inflammation by increasing expression of the deubiquitinase CYLD
}

Kensei Komatsu ${ }^{1, \star}$, Ji-Yun Lee ${ }^{1, \star}$, Masanori Miyata', Jae Hyang Lim², Hirofumi Jono ${ }^{3}$, Tomoaki Koga ${ }^{4}$, Haidong $\mathrm{Xu}^{1}$, Chen $\mathrm{Yan}^{5}$, Hirofumi Kai ${ }^{6} \&$ Jian-Dong $\mathrm{Li}^{1}$

The deubiquitinase CYLD acts as a key negative regulator to tightly control overactive inflammation. Most anti-inflammatory strategies have focused on directly targeting the positive regulator, which often results in significant side effects such as suppression of the host defence response. Here, we show that inhibition of phosphodiesterase 4B (PDE4B) markedly enhances upregulation of CYLD expression in response to bacteria, thereby suggesting that PDE4B acts as a negative regulator for CYLD. Interestingly, in Cyld-deficient mice, inhibition of PDE4B no longer suppresses inflammation. Moreover, PDE4B negatively regulates CYLD via specific activation of JNK2 but not JNK1. Importantly, ototopical post-inoculation administration of a PDE4 inhibitor suppresses inflammation in this animal model, thus demonstrating the therapeutic potential of targeting PDE4. These studies provide insights into how inflammation is tightly regulated via the inhibition of its negative regulator and may also lead to the development of new anti-inflammatory therapeutics that upregulate CYLD expression.

\footnotetext{
${ }^{1}$ Center for Inflammation, Immunity \& Infection and Department of Biology, Georgia State University, 100 Piedmont Avenue, Atlanta, Georgia 30303, USA. ${ }^{2}$ Department of Microbiology, Ewha Womans University School of Medicine, 911-1 Mok-5-dong, Yangcheon-gu, Seoul 158-710, Korea. ${ }^{3}$ Department of Clinical Pharmaceutical Sciences, Graduate School of Pharmaceutical Sciences, Kumamoto University, 1-1-1 Honjo, Kumamoto 860-8556, Japan. ${ }^{4}$ Department of Biochemistry, Juntendo University School of Medicine, 2-1-1 Hongo, Bunkyo-ku, Tokyo 113-8421, Japan. ${ }^{5}$ Aab Cardiovascular Research Institute and Department of Medicine, University of Rochester Medical Center, 601 Elmwood Avenue, Rochester, New York 14642, USA. ${ }^{6}$ Department of Molecular Medicine, Graduate School of Pharmaceutical Sciences, Kumamoto University, 5-1 Oe-Honmachi, Kumamoto 862-0973, Japan. ${ }^{\star}$ These authors contributed equally to this work. Correspondence and requests for materials should be addressed to J.-D.L. (email: jdli@gsu.edu).
} 
nflammation is a hallmark of many important human diseases including infectious diseases, chronic obstructive pulmonary diseases (COPD), otitis media (OM), asthma, arthritis, inflammatory bowel disease, atherosclerosis and cancer ${ }^{1-4}$. Although an appropriate inflammatory response is essential for eradicating pathogens, when excessive, it is clearly detrimental to the host $\mathrm{t}^{5}$. Thus, inflammation must be tightly regulated ${ }^{4,6}$. However, how this response is controlled in inflammatory diseases remains largely unknown. Moreover, despite the enormous efforts that have been put into developing antiinflammatory agents, to date, there has been limited success in developing therapies for long-term treatment of inflammatory disorders without significant side effects. Over the past decades, most strategies have focused on directly targeting the positive pathways, for example, the I $\mathrm{B} B$ kinase $\beta$ (IKK $\beta$ ), to suppress inflammation ${ }^{7}$. Although these agents often showed reasonable efficacy, they exhibited significant adverse effects, for example, increased susceptibility to infection and induction of apoptosis, which prevented their further clinical use ${ }^{8,9}$. Thus, there is an urgent need for developing novel therapeutic strategies without causing serious side effects by avoiding the direct targeting of the positive regulators of inflammation.

In comparison with the positive regulators of inflammation, for example, IKK $\beta$, the negative regulators, in particular inducible negative feedback regulators, have been shown to have a critical role in its tight control, thus preventing overactive and detrimental inflammatory responses. Recent studies have identified cylindromatosis (CYLD) as a key inducible negative feedback regulator of bacteria-induced inflammation ${ }^{10,11}$. CYLD is a novel deubiquitinase and has been shown to act as a negative regulator for various signalling pathways, for example, TRAF6, NEMO and Akt by removing lysine 63-linked polyubiquitin chains from several specific substrates ${ }^{12,13}$. Mutations of CYLD can lead to the development of tumours ${ }^{14,15}$. In addition to mutations, dysregulated expression of CYLD has also been reported under various pathological conditions. For instance, the expression of CYLD is relatively low under physiological conditions but is significantly upregulated upon bacterial infections in respiratory systems $^{16-18}$. In contrast, low expression of CYLD has also been reported in tumours ${ }^{15,19}$. Interestingly, under physiological conditions, Cyld-deficient mice exhibited no overt abnormalities and have a normal lifespan ${ }^{20}$. Together, it is evident that maintaining appropriate functional activity and expression of CYLD is critical for tightly controlling overactive inflammation and cell proliferation. Thus, we hypothesized that upregulating expression of CYLD, a key negative regulator of inflammation, for example, by pharmacological inhibition of its own negative regulator, may represent a novel and advantageous antiinflammatory strategy without causing serious adverse effects often seen with targeting positive regulator of inflammation.

Phosphodiesterases (PDEs) have long been thought as attractive and excellent therapeutic targets due to their unique tissue distribution, structural and functional properties, as well as sensitivity to selective inhibitors ${ }^{21,22}$. The PDE superfamily comprises 11 subfamilies, named PDE1-PDE11 in mammals ${ }^{21,22}$. They act as important positive and negative regulators of cellular response $\mathrm{e}^{23-26}$. To date, a number of PDE inhibitors have been already successfully developed as drugs in the clinic, for example, Viagra (targeting PDE5) for erectile dysfunction and Roflumilast (targeting PDE4) for asthma and COPD ${ }^{21,27}$. However, the available general PDE4 inhibitors (targeting all of four subfamily members A-D) exhibit serious, sometimes intolerable, adverse effects, for example, emesis, due to its inhibitory effect on PDE4D 21,28,29. Moreover, inhibition of PDE4D caused impaired growth $^{30}$. Thus, identifying another PDE4 subfamily member, for example, PDE4B but not PDE4D, as a key regulator and therapeutic target for inflammation may lead to the development of a novel and more tolerable anti-inflammatory agent for treating inflammatory disorders such as OM, a prevalent childhood disease.

In the present study, we identified PDE4B as a key negative regulator for CYLD via selective activation of c-jun $\mathrm{N}$-terminal kinase 2 (JNK2), but not JNK1, and inhibition of PDE4B significantly enhanced NTHi-induced CYLD expression and suppressed inflammation. These studies provide insights into the molecular mechanisms underlying the tight regulation of inflammation via inhibition of its negative regulator and may lead to the development of new anti-inflammatory therapeutics via upregulating CYLD expression.

\section{Results}

PDE4 is a key negative regulator for upregulation of CYLD. PDEs have long been thought of as excellent therapeutic targets $^{21,27}$. As PDE4 has been shown to have a critical role in mediating immune response, we initially sought to determine whether PDE4 acts as a key negative regulator for CYLD expression. We first evaluated the effects of specific PDE4 inhibitors on the upregulation of CYLD induced by the bacterial pathogen NTHi (clinical strain 12). Interestingly, pharmacologic inhibition of PDE4 using specific PDE4 inhibitor Rolipram markedly enhanced NTHi-induced upregulation of CYLD at both messenger RNA and protein levels in human lung A549, human middle ear HMEEC and human primary bronchial NHBE cells cultured under both conventional and air-liquid interface culture conditions (Fig. 1a-d). Similarly, Rolipram also enhanced upregulation of CYLD at mRNA and protein levels in the lung and the middle ear mucosa of well-established mouse models of lung inflammation and middle ear inflammation (OM) as assessed by performing real-time quantitative PCR (Q-PCR) and immunofluorescence (IF) staining using CYLD-specific antibody (Fig. 1e-h). In addition to Rolipram, Roflumilast, the clinically approved PDE4-specific inhibitor, also significantly enhanced NTHi-induced upregulation of CYLD (Fig. 1i). It should be noted that similar results were also observed in the cells treated with three additional commonly used clinical NTHi strains 1479, 2019 and 5657, demonstrating the generalizability of the negative role of PDE4 in regulating CYLD induction by multiple NTHi bacterial strains (Supplementary Fig. S1). Together, our data suggest that PDE4 acts as a key negative regulator of CYLD induction by multiple NTHi strains in human epithelial cells in vitro and in mouse models of both lung and middle ear inflammation.

Inhibition of PDE4 suppresses NTHi-induced inflammation. We next determined whether PDE4 inhibitor suppresses NTHiinduced inflammation. As expected, the PDE4 inhibitor Rolipram potently inhibited NTHi-induced nuclear factor- $\kappa \mathrm{B}(\mathrm{NF}-\kappa \mathrm{B})$ activation (Fig. 2a) and upregulation of key pro-inflammatory mediators including interleukin (IL)-1 $\beta$, IL-8 and tumour necrosis factor- $\alpha$ (TNF- $\alpha$ ) in A549, HMEEC and primary NHBE cells as assessed by luciferase assay and Q-PCR (Fig. $2 b$ and Supplementary Fig. S2a,b). Consistently with its anti-inflammatory effects in vitro, intraperitoneal (i.p.) preadministration of Rolipram also inhibited NTHi-induced upregulation of key proinflammatory mediators including MIP-2 (mouse homologue of human IL-8) in the lung and the middle ear of mice (Fig. 2c,d). Moreover, histopathological analysis revealed that Rolipram inhibited NTHi-induced inflammation in both lung and middle ear of mice (Fig. 2e,f). In addition, Rolipram also inhibited the middle ear mucosal thickening (Fig. 2g) and the typical otoscopic pathological changes of OM including congestion and swelling of 

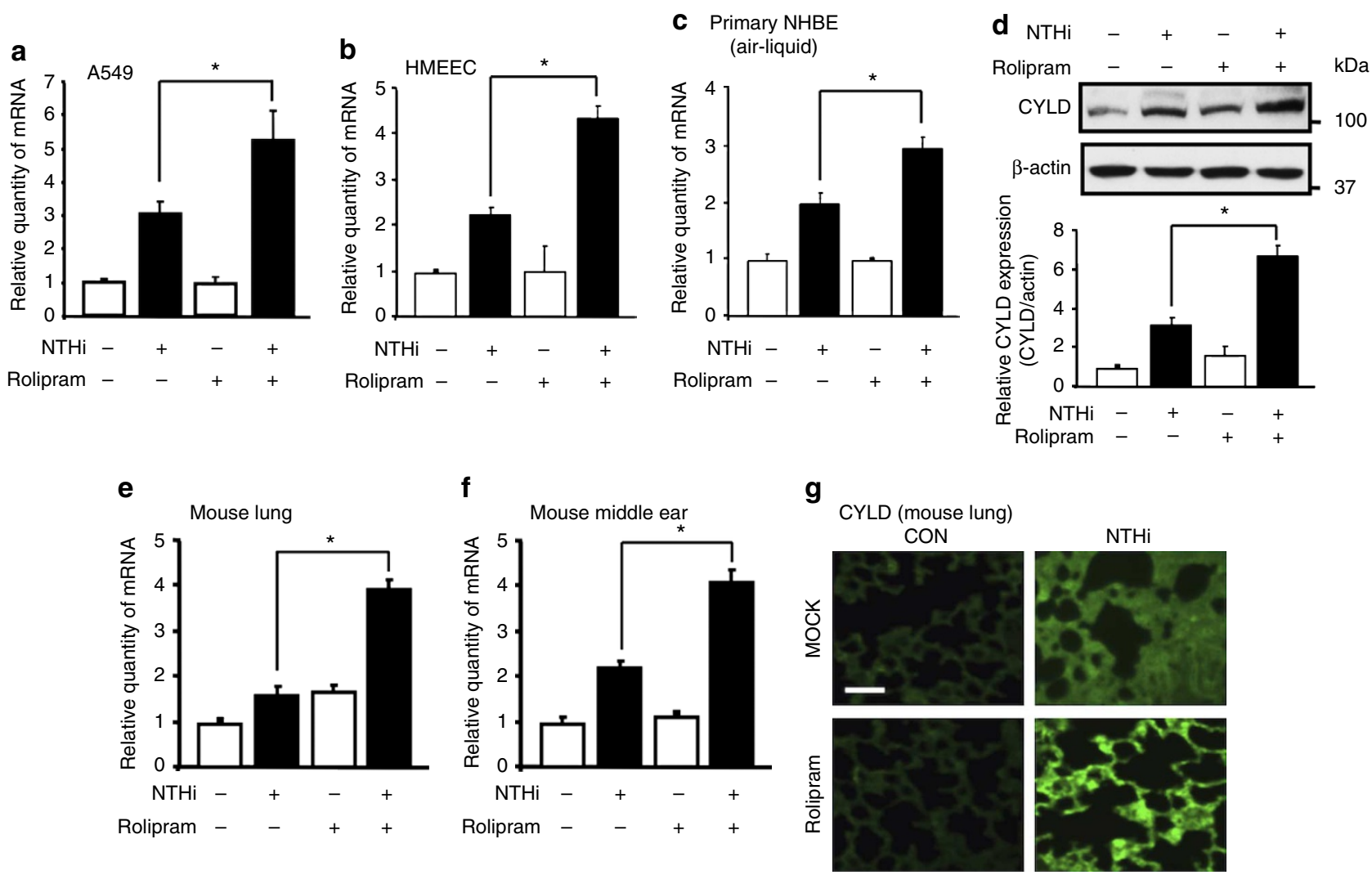

h
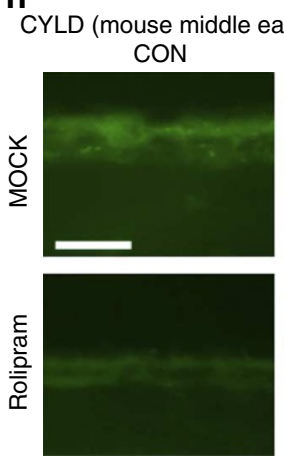

$\mathrm{NTHi}$

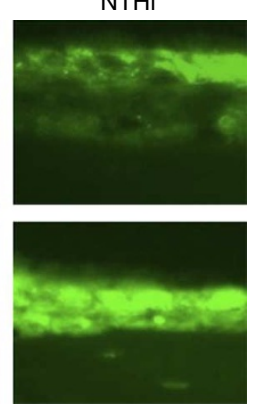

i In vitro (HMEEC)

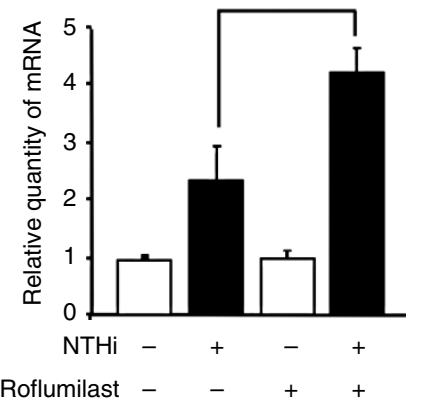

In vivo (mouse middle ear)

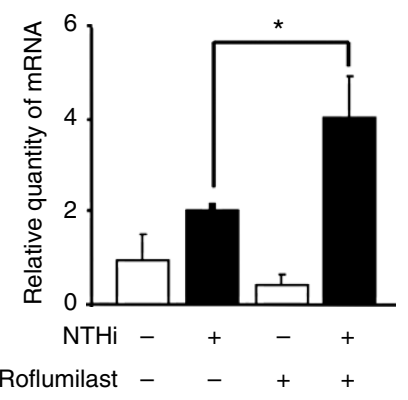

Figure 1 | PDE4 acts as a key negative regulator for NTHi-induced upregulation of CYLD expression. (a-c) CYLD mRNA expression was measured in (a) human lung A549, (b) human middle ear HMEEC or (c) human primary bronchial NHBE cells pretreated with Rolipram (10 $\mu \mathrm{M})$ for $1 \mathrm{~h}$ and stimulated with NTHi for $5 \mathrm{~h}$. (d) HMEEC cells were pretreated with Rolipram for $1 \mathrm{~h}$, followed by NTHi stimulation, and cell lysates were analysed by immunoblotting with the indicated antibodies (upper panel). CYLD protein expression was quantified from three independent experiments (lower panel). CYLD mRNA expression was measured in (e) lung and (f) middle ear tissues from C57BL/6J mice preinoculated with Rolipram (10 mg/kg) for $2 \mathrm{~h}$ and inoculated with NTHi for $9 \mathrm{~h}$. Mouse tissues from (g) lung and (h) middle ear were stained against CYLD after NTHi inoculation, with or without inoculation of Rolipram (magnification $\times 200$ in $\mathbf{g}$; $\times 400$ in h, respectively). Scale bars, $20 \mu \mathrm{m}$. (i) CYLD mRNA expression in HMEEC (left panel) or mouse middle ear tissues (right panel) was measured after NTHi treatment, pretreated with or without Roflumilast ( $1 \mu \mathrm{M}$ for in vitro, $5 \mathrm{mg} / \mathrm{kg}$ for in vivo). Data in a-f and $\mathbf{i}$ are mean \pm s.d. $(n=3) .{ }^{\star} P<0.05$. Statistical analysis was performed using Student's $t$-test. Data are representative of three or more independent experiments.

the tympanic membrane and mucous effusion accumulation inside the bulla, as assessed by performing otoscopic examination in NTHi-inoculated mice (Fig. 2h). Similarly to Rolipram, Roflumilast, also significantly inhibited NTHi-induced inflammation in vitro and in vivo (Fig. 2i and Supplementary Fig. S2c-e). Collectively, these data demonstrate that inhibition of PDE4 indeed suppressed NTHi-induced inflammatory response in human lung and middle ear epithelial cells in vitro and also in well-established mouse models of lung and middle ear inflammation.
PDE4 inhibition suppresses inflammation by inducing CYLD. Having demonstrated that inhibition of PDE4 enhanced upregulation of CYLD and suppressed NTHi-induced inflammation, it is still unclear whether inhibition of PDE4 suppresses inflammation via upregulating the expression of CYLD, a key negative regulator of inflammation, or via inhibiting key positive regulators of inflammation, for example, IKK $\beta$. We first determined if PDE4 inhibitor still suppresses NTHi-induced inflammation in CYLD-depleted cells. Interestingly, PDE4 inhibitor Rolipram no longer inhibited NTHi-induced upregulation of 
a

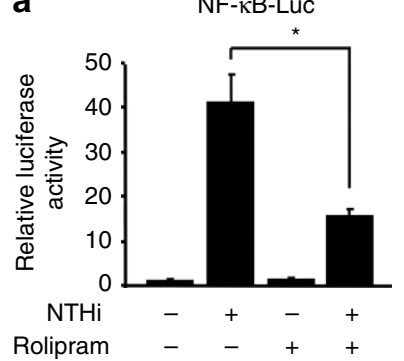

b

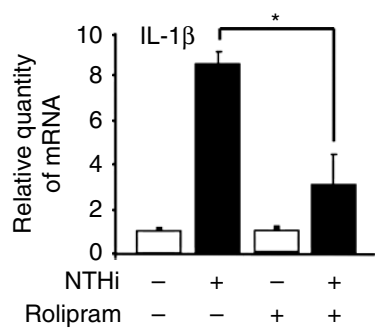

A549

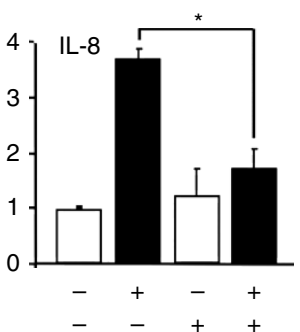

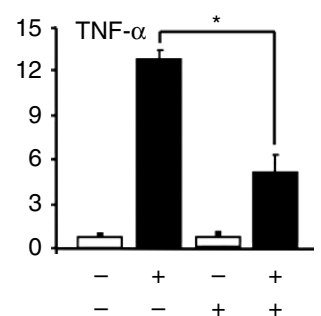

C

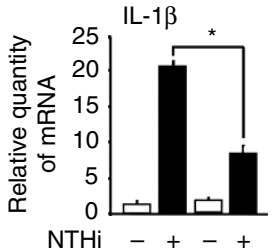

Mouse lung

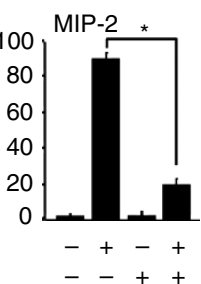

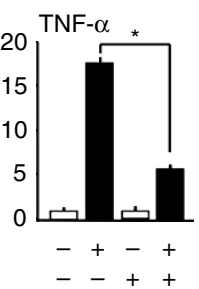

d

d $\quad$ Mouse middle ear

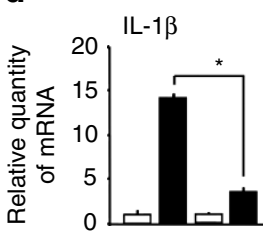

$\mathrm{NTHi}-+-+$ Rolipram - - + +
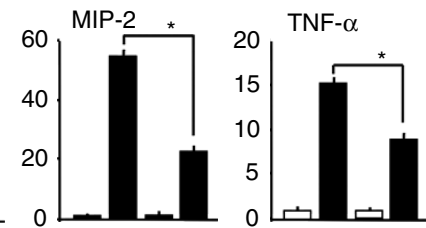

-++
-+
Rolipram - - + +

f

e

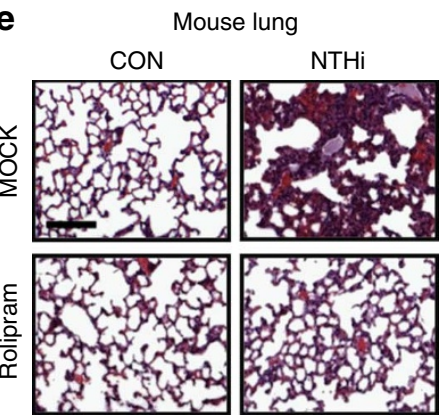

h
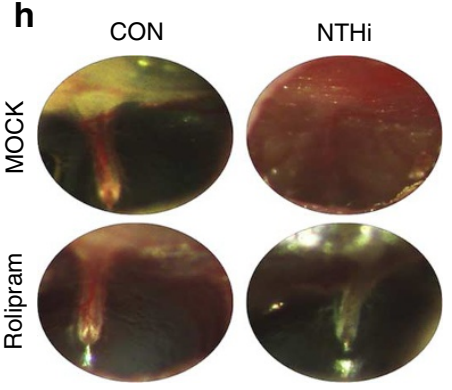
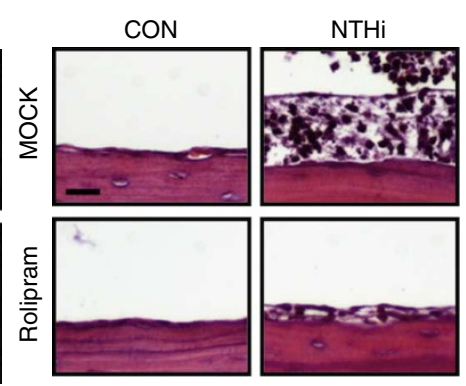

g
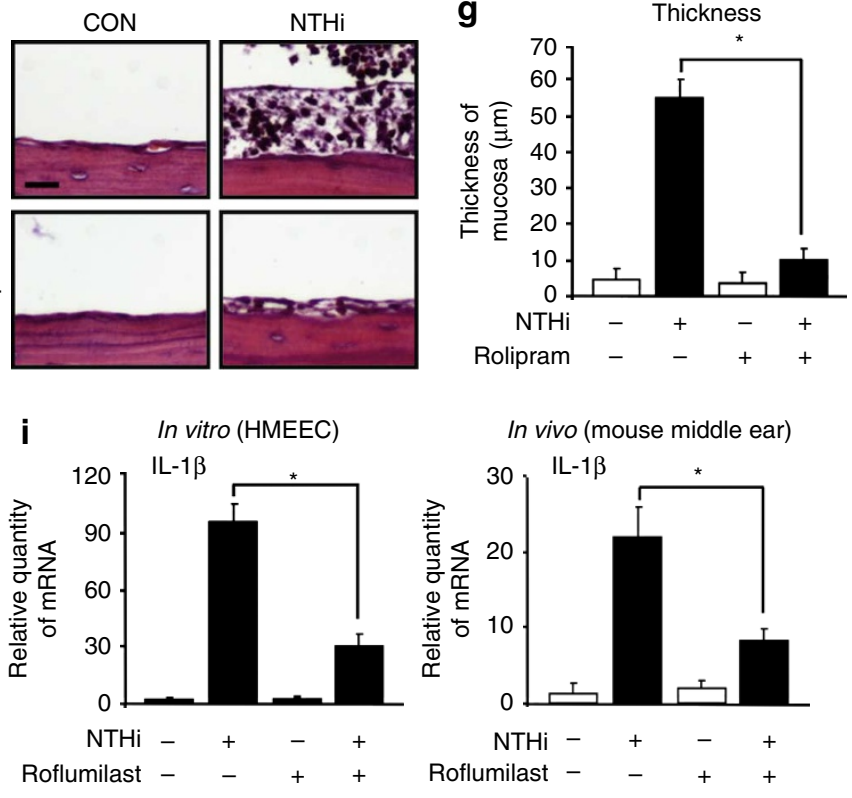

Figure 2 | Inhibition of PDE4 leads to suppression of NTHi-induced inflammation in vitro and in vivo. (a) A549 cells were pretreated with Rolipram $(10 \mu \mathrm{M})$ for $1 \mathrm{~h}$, followed by NTHi stimulation, and NF- $\mathrm{KB}$-promoter activity was determined. (b) mRNA expression of pro-inflammatory cytokines was measured in A549 cells pretreated with Rolipram for $1 \mathrm{~h}$ and stimulated with NTHi. mRNA expression of pro-inflammatory cytokines was measured in (c) lung and (d) middle ear tissues from C57BL/6J mice preinoculated with Rolipram (10 mg/kg) for $2 \mathrm{~h}$ and inoculated with NTHi for $9 \mathrm{~h}$. Haematoxylin and eosin staining of (e) lung and (f) middle ear tissues from C57BL/6J mice preinoculated with Rolipram for $2 \mathrm{~h}$ and inoculated with NTHi for $9 \mathrm{~h}$ (magnification $\times 100$ in $\mathbf{e} ; \times 400$ in $\mathbf{f}$, respectively). Scale bars, $100 \mu \mathrm{m}$ in $\mathbf{e} ; 20 \mu \mathrm{m}$ in $\mathbf{f}$. (g) Thickness of middle ear mucosa in C57BL/6J mice preinoculated with Rolipram and inoculated with NTHi was measured from 15 middle ear tissue sections per experimental group. (h) Mice were transtympanically inoculated with NTHi with or without Rolipram pretreatment, and tympanic cavity was observed and recorded under the otoscope. (i) IL-1 $\beta$ mRNA expression in HMEEC (left panel) or mouse middle ear tissues (right panel) was measured after NTHi treatment pretreated with or without Roflumilast ( $1 \mu \mathrm{M}$ for in vitro, $5 \mathrm{mg} \mathrm{kg}^{-1}$ for in vivo). Data in $\mathbf{a}-\mathbf{d}, \mathbf{g}$ and $\mathbf{i}$ are mean \pm s.d. ( $n=3$ in $\mathbf{a}-\mathbf{d}, \mathbf{i}$ and 15 in $\mathbf{g}$ ). ${ }^{\star} p<0.05$. Statistical analysis was performed using Student's t-test. CON, control. Data are representative of three or more independent experiments.

pro-inflammatory mediators in A549 and HMEEC cells in which CYLD was depleted with CYLD short interfering RNA (Fig. 3a and Supplementary Fig. S3a). Similarly, Rolipram also failed to inhibit NTHi-induced mRNA expression of these cytokines in Cyld-deficient mouse embryonic fibroblasts (MEFs) (Fig. 3b). Moreover, similar results were also confirmed in mouse models of NTHi-induced inflammation in the lung and middle ear. As shown in Fig. 3c,d, preadministration (i.p.) of Rolipram no longer inhibited NTHi-induced upregulation of TNF- $\alpha$ and IL-1 $\beta$ in the lung and middle ear of Cyld-deficient mice. As revealed by performing histopathological analysis, Rolipram also failed to suppress NTHi-induced inflammatory response in Cyld-deficient mice as compared with wild-type (WT) mice (Fig. 3e,f). In agreement with these findings, Rolipram also no longer inhibited polymorphonuclear neutrophil infiltration in bronchoalveolar lavage fluids and the middle ear mucosal thickening in 
a

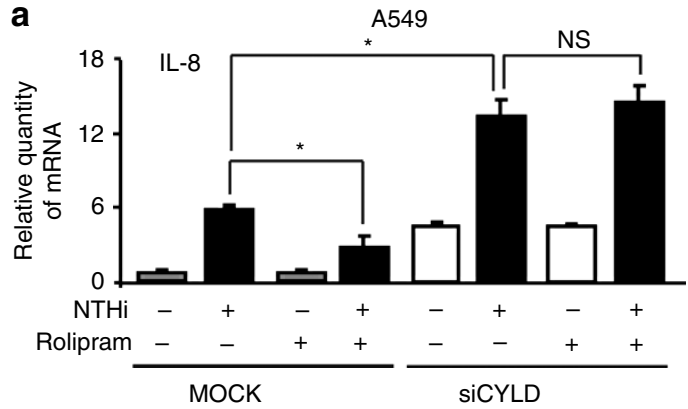

C

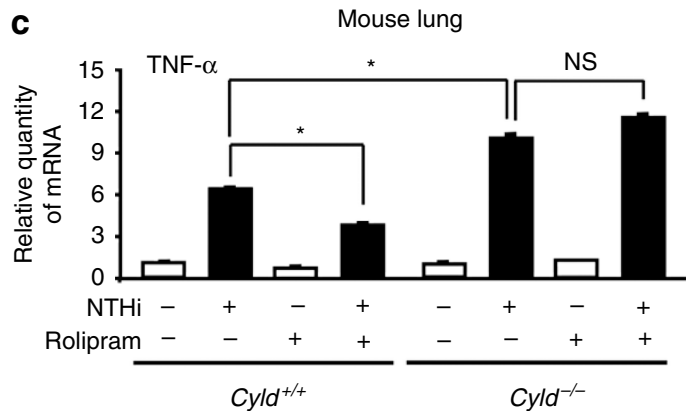

e

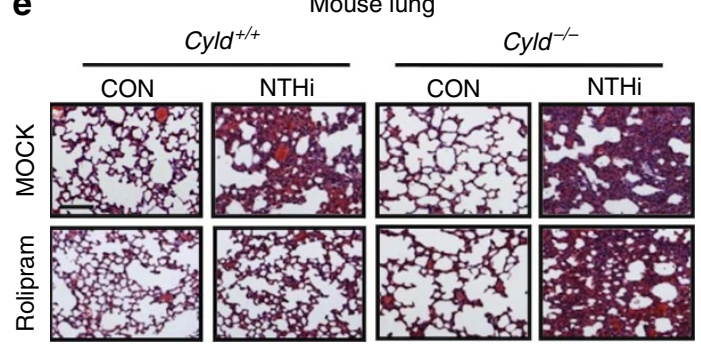

g

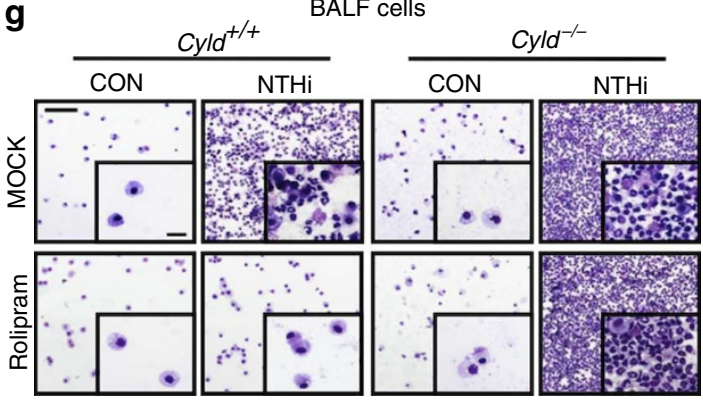

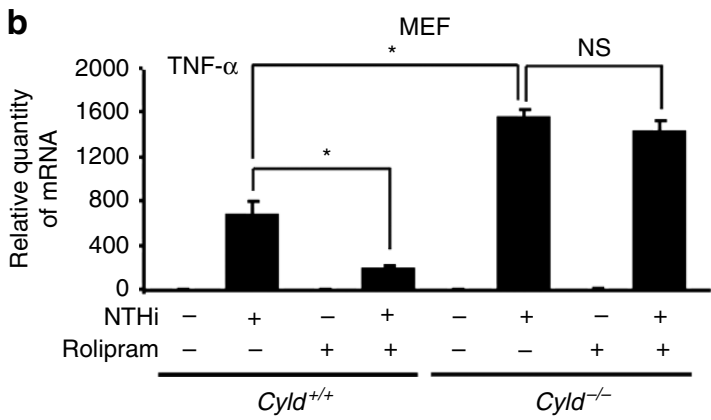

d

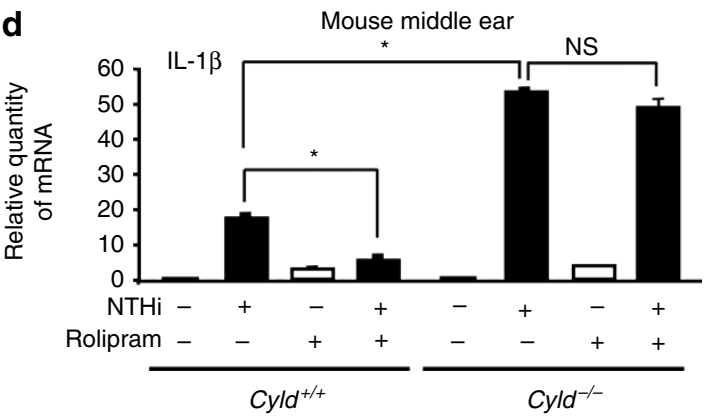

$\mathbf{f}$

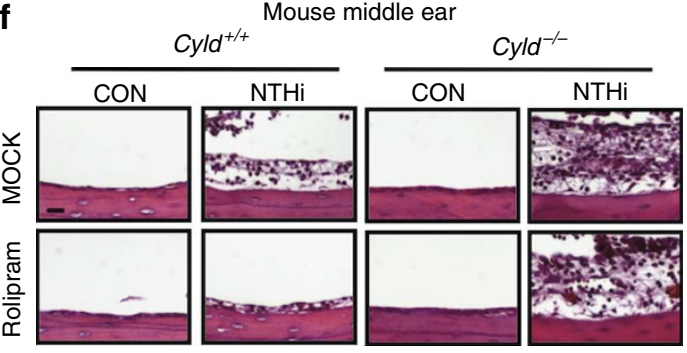

h

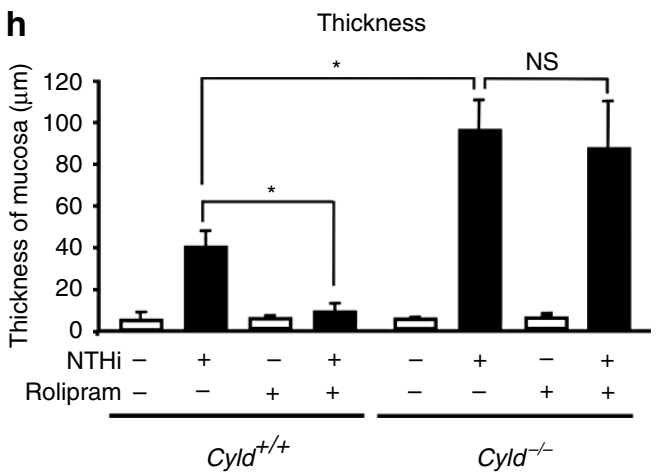

Figure 3 | Inhibition of PDE4 leads to suppression of NTHi-induced inflammation via upregulation of CYLD expression. (a) A549 cells transfected with siCON or siCYLD were pretreated with Rolipram $(10 \mu \mathrm{M})$ for $1 \mathrm{~h}$, and IL-8 mRNA expression was measured post NTHi treatment. (b) TNF- $\alpha$ mRNA expression was measured in MEF cells from Cyld ${ }^{+/+}$and Cyld ${ }^{-/-}$mice pretreated with Rolipram $(10 \mu \mathrm{M})$ for $1 \mathrm{~h}$ and stimulated with NTHi. mRNA expression of pro-inflammatory cytokines was measured in (c) lung and (d) middle ear tissues from Cyld ${ }^{+}+$and Cyld ${ }^{-/-}$mice preinoculated with Rolipram (10 $\mathrm{mg} \mathrm{kg}^{-1}$, i.p.) for $2 \mathrm{~h}$ and inoculated with NTHi. Haematoxylin and eosin staining of (e) lung and (f) middle ear tissues from Cyld $+/+$ and Cyld $^{-/-}$mice preinoculated with Rolipram for $2 \mathrm{~h}$ and inoculated with NTHi (magnification $\times 100$ in $\mathbf{e} ; \times 400$ in $\mathbf{f}$, respectively). Scale bars, $100 \mu \mathrm{m}$ in $\mathbf{e}$; $20 \mu \mathrm{m}$ in f. (g) Polymorphonuclear neutrophils (PMNs) were collected from the lung tissues of Cyld ${ }^{+} /+$and Cyld ${ }^{-/-}$mice preinoculated with Rolipram and inoculated with NTHi by performing bronchoalveolar lavage. PMNs were stained with Diff-Quik staining system after cytocentrifugation (magnification $\times 100$ in large frame; $\times 400$ in inserted frame). Scale bars, $100 \mu \mathrm{m}$ in large frame; $200 \mu \mathrm{m}$ in inserted frame. (h) Thickness of middle ear mucosa in $\mathrm{Cyld}^{+/+}$and Cyld ${ }^{-/-}$mice preinoculated with Rolipram and inoculated with NTHi was measured from 15 middle ear tissue sections per experimental group. Data in a-d and $\mathbf{h}$ are mean \pm s.d. ( $n=3$ in $\mathbf{a}-\mathbf{d}$ and 15 in $\mathbf{h}$ ). ${ }^{\star} P<0.05$. Statistical analysis was performed using Student's $t$-test. Data are representative of three or more independent experiments. CON, control; NS, nonsignificant.

Cyld-deficient mice as compared with WT mice (Fig. 3g,h and Supplementary Fig. S3b,c). In addition to Rolipram, Roflumilast also no longer inhibited NTHi-induced upregulation of proinflammatory mediators in HMEEC and A549 cells in which
CYLD was depleted with CYLD siRNA (Supplementary Fig. S3d,e). These data suggest that inhibition of PDE4 indeed suppressed NTHi-induced inflammation in vitro and in vivo via upregulating CYLD expression. 
We next determined whether PDE4 inhibitors may also suppress inflammation via directly inhibiting $\mathrm{IKK} \beta$, the key positive regulator of NF- $\mathrm{BB}$-dependent inflammation, or $\mathrm{p} 65$, the key subunit of NF- $\kappa$ B. As shown in Supplementary Fig. S4a,b, Rolipram did not significantly inhibit activation of NF- $\kappa B$ when induced by overexpressing either a constitutively active form of IKK $\beta$ or WT p65. Taken together, our data confirmed our hypothesis that PDE4 acts as a crucial inhibitor for CYLD, a key negative regulator for inflammation. Additionally, we also showed that inhibition of PDE4 using PDE4-specific inhibitors suppresses NTHi-induced inflammation by upregulating expression of CYLD, but not by inhibiting IKK $\beta$ or NF- $\kappa \mathrm{B}$ p 65 , the positive regulators of inflammation.

PDE4B mediates inflammation by downregulating CYLD. We next sought to determine which PDE4 subfamily member is specifically involved in mediating NTHi-induced inflammation by negatively regulating CYLD. PDE 4 consists of four subfamily genes, $P D E 4 A$ to $D$, encoding Rolipram-sensitive PDEs ${ }^{27}$. PDE4 exerts its cellular functions by catalysing and degrading cyclic AMP (cAMP), one of the important second messengers in regulating numerous pathological processes in response to stimulants, for example, bacterial pathogens ${ }^{22}$. Previous studies indicate that upregulation of PDE4 has an important role in modulating pathological responses ${ }^{28,31,32}$. Thus, we first determined whether PDE4 is upregulated by NTHi. As shown in Fig. 4a,b and Supplementary Fig. S5a,b, PDE4B is selectively and markedly upregulated by NTHi at mRNA and protein levels in HMEEC, A549 and primary NHBE cells, in vitro. Similar to the in vitro findings, NTHi-induced upregulation of PDE4B at both mRNA and protein levels was also confirmed in the mouse lung and middle ear in vivo as assessed by performing Q-PCR and IF analysis (Fig. 4c-f). Consistent with these results, PDE4B a

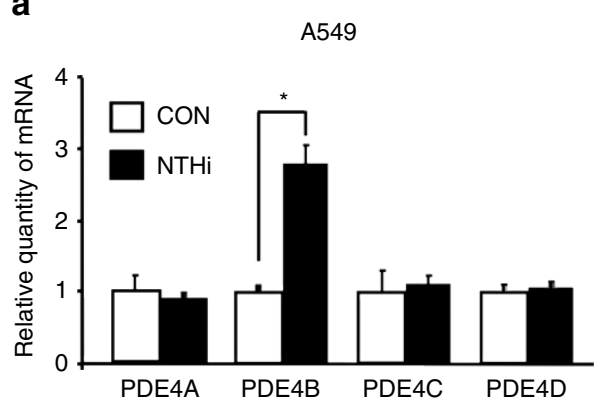

d Mouse middle ear

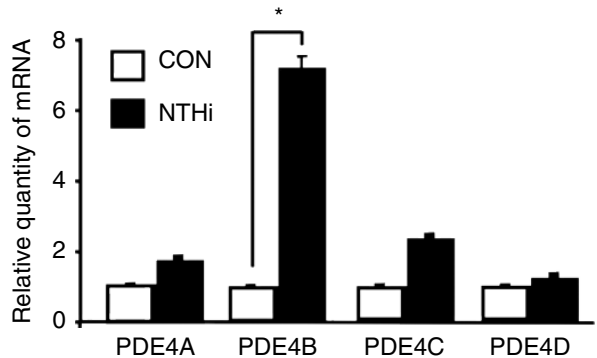

g

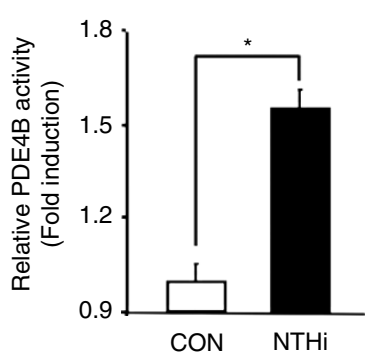

h

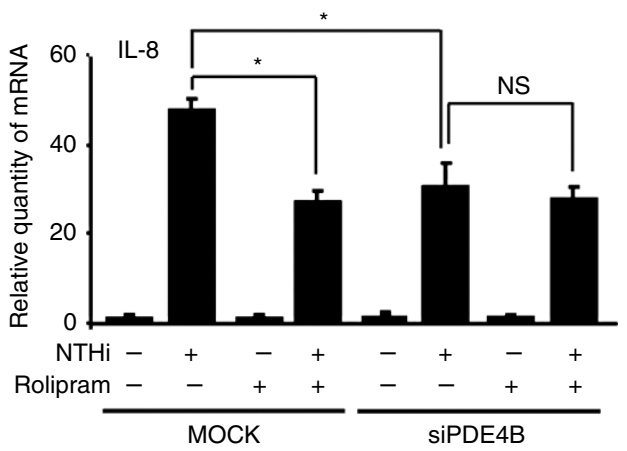

e
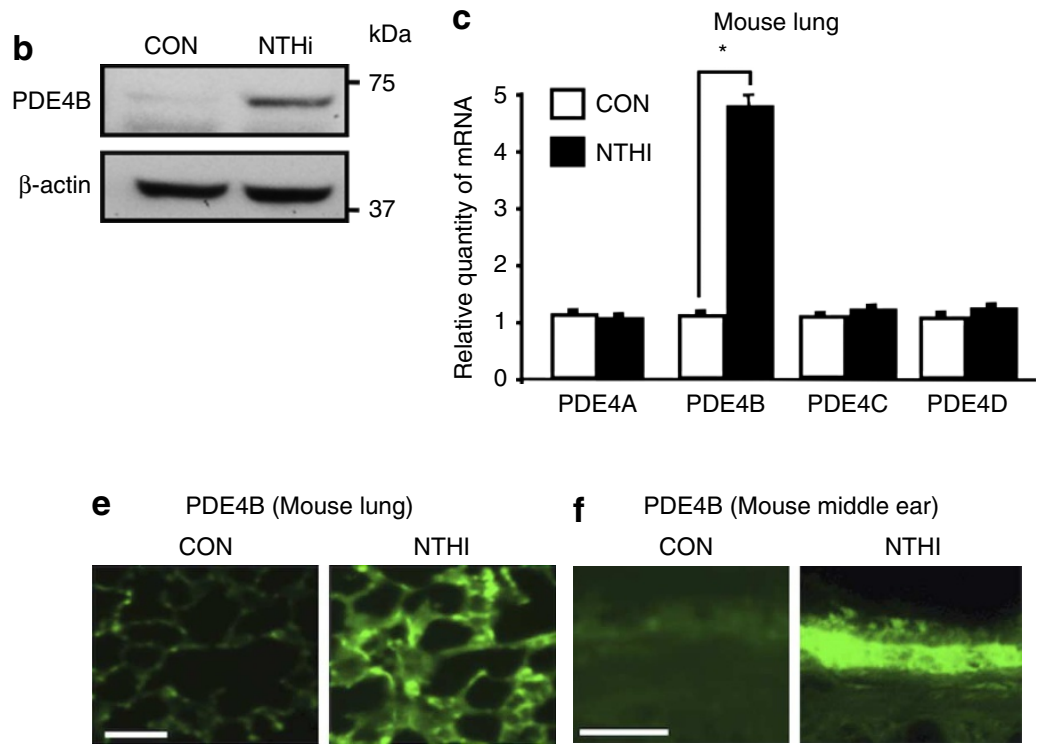

f PDE4B (Mouse middle ear)
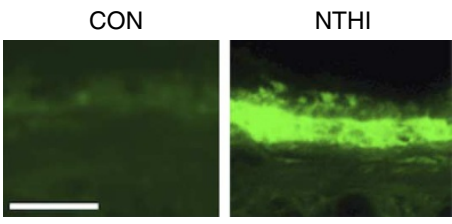

i

A549

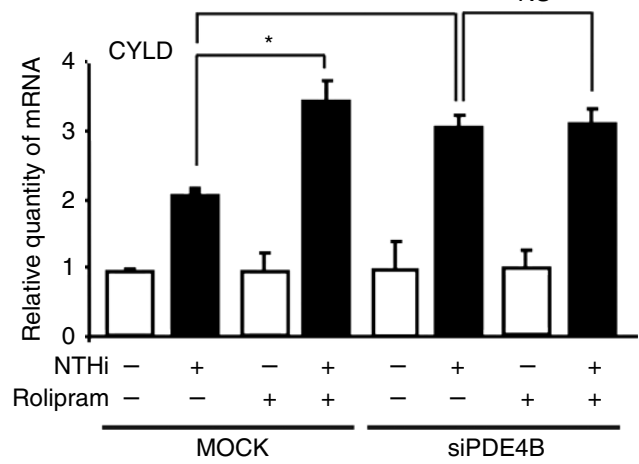

Figure 4 | PDE4B has a crucial role in mediating NTHi-induced inflammation by negatively regulating CYLD expression. (a) PDE4 mRNA expression was measured in A549 cells stimulated with NTHi for $1.5 \mathrm{~h}$. (b) Cells were treated with NTHi, and cell lysates were analysed by immunoblotting with the indicated antibodies. PDE4 mRNA expression was measured in (c) lung and (d) middle ear tissues from C57BL/6J mice inoculated with NTHi for $4 \mathrm{~h}$. Mouse tissues from (e) lung and (f) middle ear were stained against PDE4B after NTHi inoculation (magnification $\times 200$ in $\mathbf{e}$; $\times 400$ in $\mathbf{f}$, respectively). Scale bars, $20 \mu \mathrm{m}$. (g) Lysates from cells treated with NTHi were immunoprecipitated with anti-PDE4B antibody, and analysed by PDE activity assay. (h) Cells transfected with siCON or siPDE4B were pretreated with Rolipram $(10 \mu \mathrm{M})$ for $1 \mathrm{~h}$, and IL-8 mRNA expression was measured post NTHi treatment. (i) A549 cells transfected with siCON or siPDE4B were pretreated with Rolipram for $1 \mathrm{~h}$, and CYLD mRNA expression was measured post NTHi treatment. Data in $\mathbf{a}, \mathbf{c}, \mathbf{d}$ and $\mathbf{g}$-i are mean \pm s.d. $(n=3) .{ }^{\star} P<0.05$. Statistical analysis was performed using Student's $t$-test. CON, control. Data are representative of three or more independent experiments. NS, nonsignificant. 
enzymatic activity was also upregulated by NTHi (Fig. 4g). Together, these data suggest that PDE4B may have an important role in mediating NTHi-induced inflammation via negative regulation of CYLD.

We next determined whether PDE4B has an important role in mediating NTHi-induced inflammation by negatively regulating CYLD. We first investigated whether PDE4 inhibitor Rolipram still suppresses NTHi-induced inflammation in PDE4B-depleted cells. As shown in Fig. 4h and Supplementary Fig. S5c-e, Rolipram no longer further inhibited NTHi-induced upregulation of pro-inflammatory mediator IL- $1 \beta$ and IL-8 in HMEEC and A549 cells in which PDE4B was depleted with PDE4B siRNA. Similarly, Rolipram also failed to further enhance NTHi-induced CYLD expression in PDE4B-depleted cells (Fig. 4i and Supplementary Fig. S5f). Taken together, our data suggest that PDE4B indeed has a crucial role in mediating NTHi-induced inflammation by negatively regulating CYLD expression.

PDE4B downregulates CYLD via activation of JNK2 pathway. We next sought to determine how PDE4B mediates NTHiinduced inflammation via negatively regulating CYLD expression. As the mitogen-activated protein kinase (MAPK) JNK has an important role in mediating bacteria-induced host response ${ }^{33-35}$, we initially determined the involvement of JNK in regulating CYLD using specific JNK inhibitor, SP600125. As shown in Fig. 5a,b, inhibition of JNK using SP600125 markedly enhanced NTHi-induced upregulation of CYLD and suppressed inflammation in HMEEC cells in vitro and in the middle ear of mouse in vivo. Interestingly, PDE4 inhibition using Rolipram no longer enhanced NTHi-induced upregulation of CYLD and suppressed inflammation in HMEEC cells that had been already pretreated with SP600125 (Fig. 5c). These results suggest that PDE4B negatively regulates CYLD expression and mediates inflammation via the JNK pathway.

As JNK1 and 2 represent the two major isoforms expressed in the middle ear and lung epithelial cells, we next determined which isoform is involved. Interestingly, PDE4 inhibitor selectively suppressed NTHi-induced activation of JNK2, but not JNK1 (Fig. 5d). We further determined whether PDE4B mediates the selective activation of JNK2 induced by NTHi using siRNA PDE4B. As shown in Fig. 5e, PDE4B knockdown selectively inhibited activation of JNK2 but not JNK1, thereby confirming that PDE4B negatively regulates NTHi-induced CYLD expression and mediates inflammation via specific activation of JNK2 but not JNK1.

Having established that JNK2 acts downstream of PDE4B in negatively regulating CYLD expression and mediating NTHiinduced inflammation, we sought to determine whether JNK2 indeed acts upstream of CYLD to mediate PDE4B-dependent NTHi-induced inflammation. As shown in Fig. 5f and Supplementary Fig. S6, inhibition of PDE4 using Rolipram still enhanced NTHi-induced upregulation of CYLD in JNK1depleted cells but not in JNK2-depleted cells, confirming the selective requirement of JNK2 in mediating PDE4B-depenent negative regulation of CYLD expression. We next investigated whether JNK2 pathway indeed regulates NTHi-induced inflammation in a CYLD-dependent manner using Cylddeficient cells and mice. As expected, NTHi-induced inflammation was significantly enhanced in Cyld-deficient cells and the JNK inhibitor no longer inhibited NTHi-induced inflammation in Cyld-deficient cells as compared with WT cells (Fig. 5g). Consistent with these in vitro findings, i.p. preadministration of SP600125 inhibited NTHi-induced upregulation of the key pro-inflammatory mediators in the middle ear of WT mice but not in Cyld-deficient mice (Fig. 5h).
Similarly, histopathological analysis revealed that SP600125 also failed to inhibit NTHi-induced middle ear inflammation including mucosal thickening in Cyld-deficient mice (Fig. 5i,j). Taken together, it is evident that PDE4B negatively regulates CYLD expression and mediates NTHi-induced inflammation via specific activation of JNK2 but not JNK1 pathway.

PDE4B activates JNK2 by inhibiting cAMP-PKA pathway. PDE4 exerts its effects by catalysing and degrading cAMP and thereby tightly controlling its intracellular concentrations ${ }^{21}$. Inhibition of PDE4 activity leads to an increase in intracellular concentration of cAMP. Thus, we sought to determine whether increased cAMP does have an important role in inhibiting JNK2 activation, which in turn enhances CYLD expression and suppresses inflammation. We first evaluated the effect of 8bromo-cAMP (8-Br-cAMP), a cell-permeable cAMP analogue which is more resistant to PDE4 than cAMP, on NTHi-induced JNK activation. Similarly to the effect of PDE4 inhibition, 8-BrcAMP selectively inhibited NTHi-induced activation of JNK2 but not JNK1 in HMEEC cells (Fig. 6a). We further confirmed the involvement of cAMP in NTHi-induced JNK2 activation by using Forskolin, a cell-permeable diterpenoid, which has been commonly used to increase levels of intracellular cAMP. As shown in Fig. 6b, Forskolin inhibited NTHi-induced activation of JNK2 but not JNK1. As protein kinase A (PKA) represents the major downstream signalling effector of cAMP, and PKA was known to be involved in regulating inflammation, we next determined whether cAMP-dependent PKA pathway is critically involved in NTHi-induced JNK2 activation by using PKA inhibitor (PKI). As shown in Fig. 6c, PKI enhanced NTHiinduced activation of JNK2 but not JNK1. Together, these data suggest that cAMP-dependent PKA pathway has an important role in mediating NTHi-induced JNK2 activation. We next determined whether cAMP-dependent PKA pathway also has a critical role in mediating NTHi-induced CYLD expression and inflammation. As shown in Fig. 6d-f, 8-Br-cAMP and Forskolin enhanced, whereas PKI inhibited, NTHi-induced upregulation of CYLD expression in HMEEC cells. Consistent with these results, 8-Br-cAMP and Forskolin inhibited, whereas PKI enhanced, NTHi-induced upregulation of IL-1 $\beta$ in HMEEC cells (Fig. $6 \mathrm{~g}-\mathrm{i}$ ). Collectively, our data suggest that PDE4B selectively mediates NTHi-induced JNK2 activation via inhibition of cAMP-PKAdependent pathway, which in turn leads to suppression of CYLD and subsequent induction of inflammation (Fig. 6j). Interestingly, the basal expression level of CYLD is elevated by Forskolin and 8Br-cAMP but not Rolipram. This discrepancy is likely due to the difference in the elevated subcellular pools of cAMP. Forskolin is known to activate a number of adenylyl cyclases and elevates a wider range of subcellular pools of cAMP. 8-Br-cAMP is a nonhydrolysable cAMP analogue resistant to PDEs. In contrast, Rolipram is a selective inhibitor for PDE4, particularly PDE4B, which hydrolyses cAMP in more restricted subcellular compartments. These results suggest the involvement of additional mechanism to induce CYLD. In addition, how CYLD mRNA expression is increased upon treatment involving cAMP, through inhibition of JNK2 or not, remains unknown. Future studies are warranted to further elucidate the underlying mechanisms by determining the effects of specific depletion of JNK1 or 2 or both in cAMP-mediated CYLD expression.

Ototopical PDE4 inhibitor suppresses inflammation. We have shown that upregulating expression of CYLD, a key negative regulator of inflammation, via inhibition of its own negative regulator, PDE4B, leads to the suppression of NTHi-induced inflammation. We have already demonstrated that specific PDE4 
a
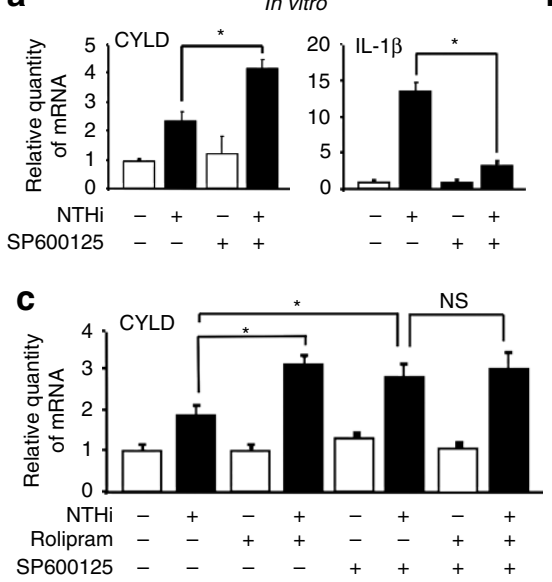

b
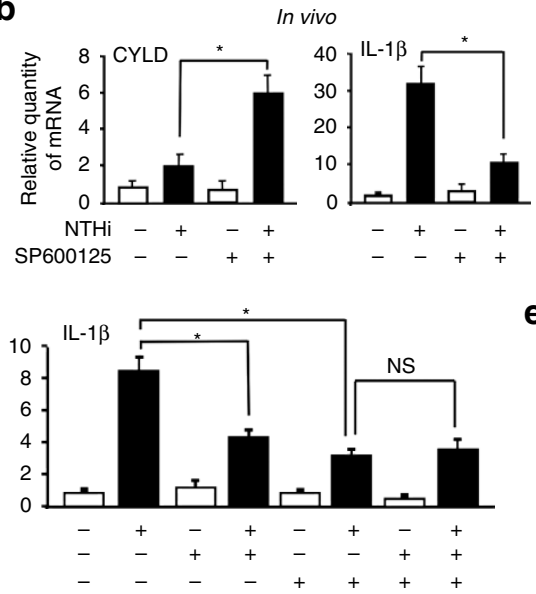

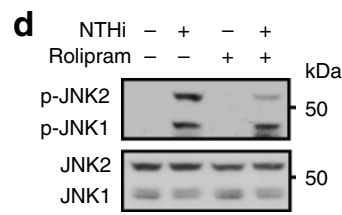

e

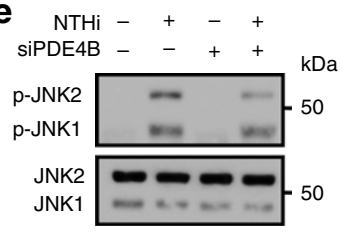

f
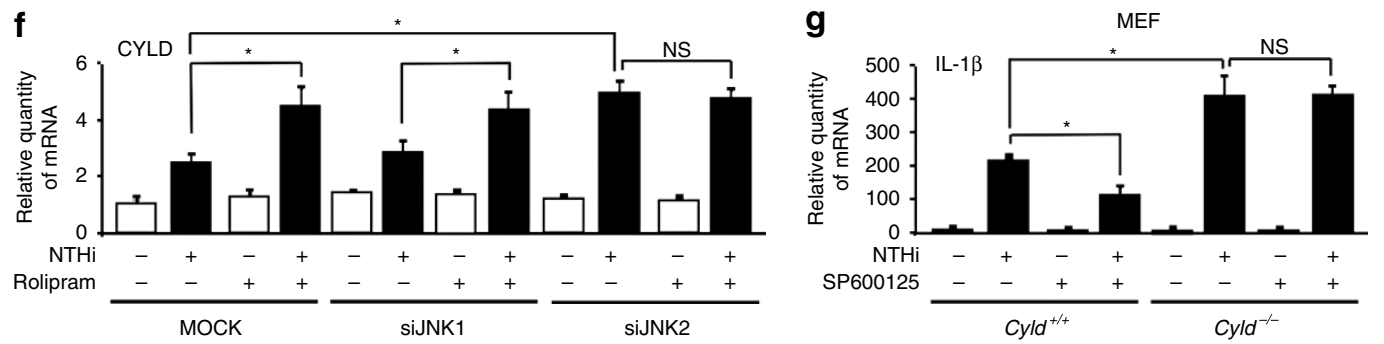

h

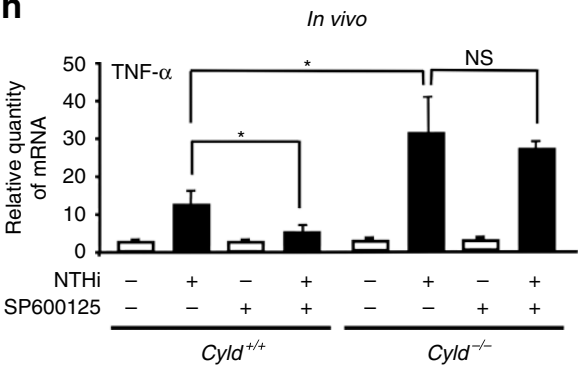

i

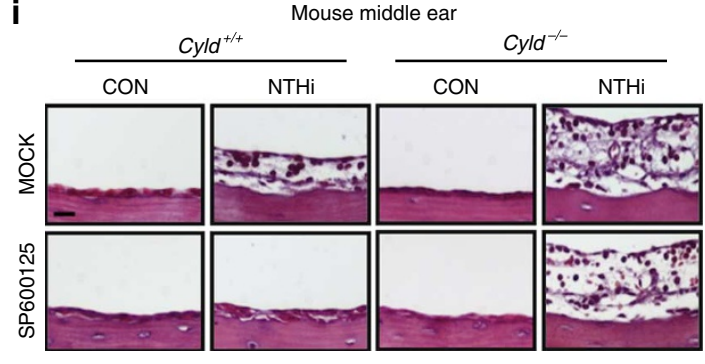

Mouse middle ear

j

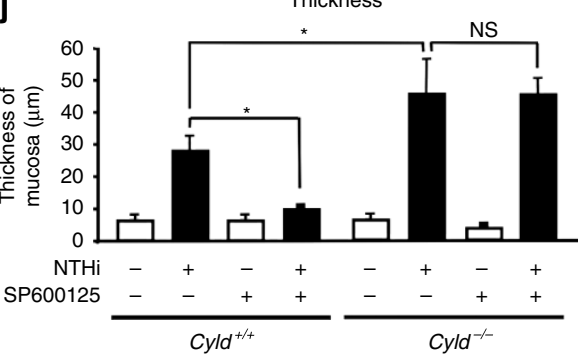


a

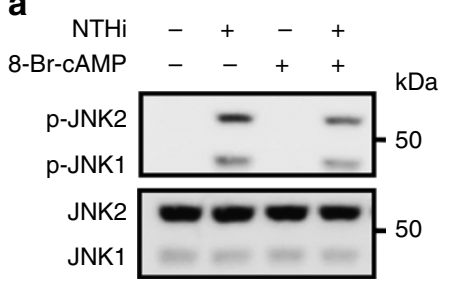

b

NTHi -+-+

Forskolin $-\quad++$

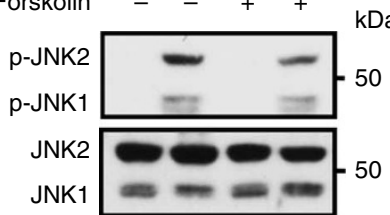

d

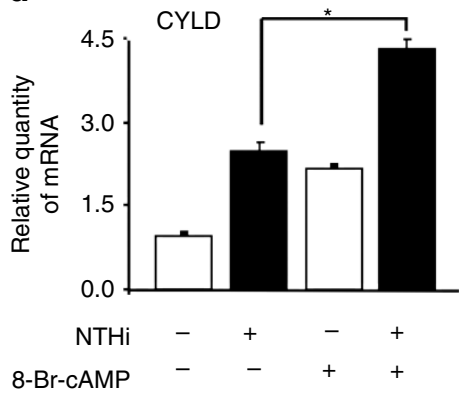

g

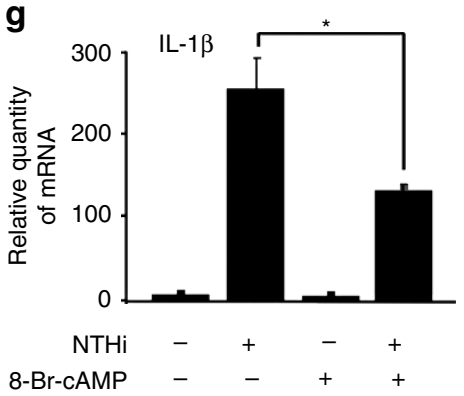

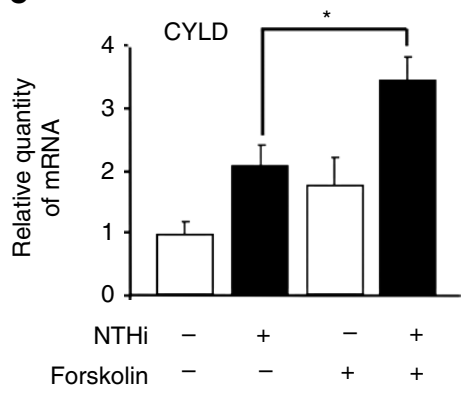

h

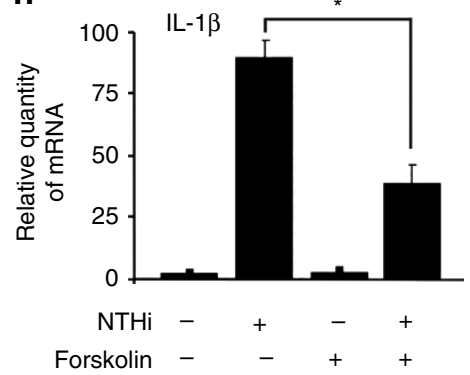

C

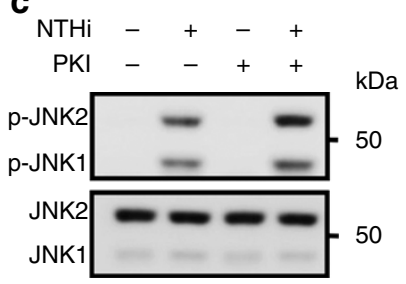

f

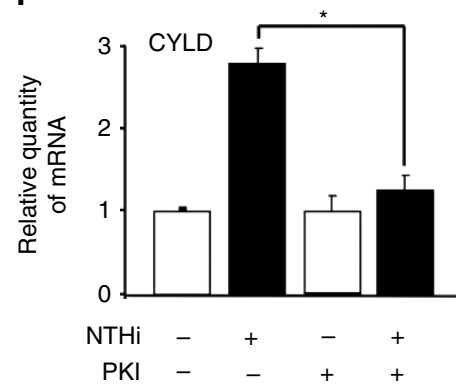

i

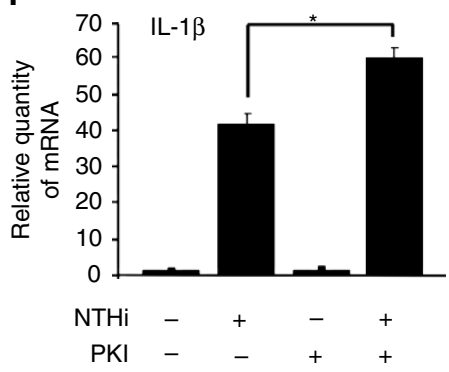

j
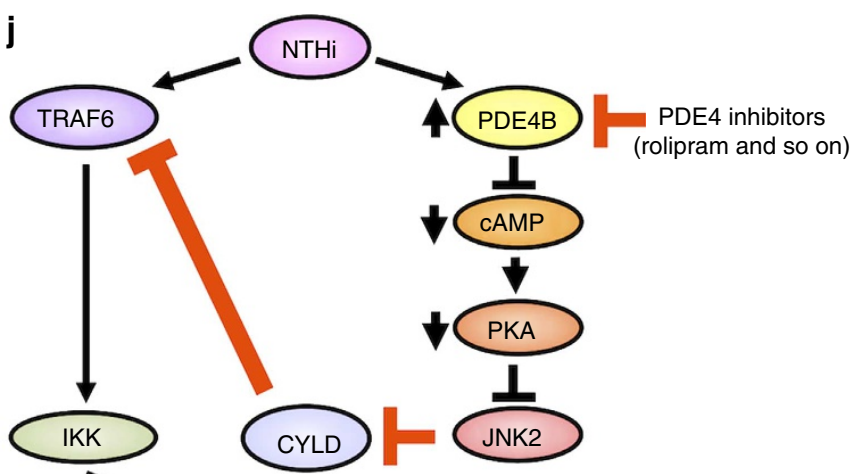

Inflammation in lung and middle ear

Figure 6 | PDE4B activates JNK2 leading to suppression of CYLD and subsequent induction of inflammation. (a-c) Cells were pretreated with (a) $100 \mu \mathrm{M} 8$-Br-cAMP, (b) $10 \mu \mathrm{M}$ Forskolin or (c) $1 \mu \mathrm{M}$ PKI for $1 \mathrm{~h}$, followed by NTHi stimulation, and cell lysates were analysed by immunoblotting with the indicated antibodies. (d-f) Cells were pretreated with (d) 8-Br-cAMP, (e) Forskolin or (f) PKI for $1 \mathrm{~h}$, followed by NTHi stimulation, and CYLD mRNA expression was measured. (g-i) Cells were pretreated with (g) 8-Br-cAMP, (h) Forskolin or (i) PKI for $1 \mathrm{~h}$, followed by NTHi stimulation, and IL-1 $\beta$ mRNA expression was measured. (j) A schematic model illustrating that PDE4B selectively mediates NTHi-induced JNK2 activation via inhibition of cAMP-PKAdependent pathway, which in turn leads to suppression of CYLD and subsequent induction of inflammation. Data in $\mathbf{d}-\mathbf{i}$ are mean \pm s.d. $(n=3)$. ${ }^{\star} P<0.05$. Statistical analysis was performed using Student's $t$-test. Data are representative of three or more independent experiments.

inhibitors, Rolipram and Roflumilast, when preadministered systemically, upregulated CYLD and suppressed subsequent inflammation in the mouse models of middle ear inflammation $(\mathrm{OM})$ and lung inflammation (Figs 1 and 2). These encouraging data thus prompted us to directly test whether post-inoculation administration of Rolipram has any therapeutic effect on treating middle ear inflammation under clinically relevant condition. Ototopical administration works effectively when the eardrum is 
perforated either pathologically or surgically by tympanostomy tube insertion ${ }^{36-38}$. Thus, we next evaluated whether ototopical administration of Rolipram upregulates CYLD and suppresses inflammation in the middle ear of mice post NTHi infection. Interestingly, both ototopical pre- and post-inoculation administration of Rolipram upregulated CYLD expression (Fig. 7a) and suppressed inflammation in a mouse OM model in vivo (Fig. $7 \mathrm{~b}-\mathrm{e}$ ). Taken together, these data demonstrate that upregulating CYLD expression via pharmacologic inhibition of PDE4 does exhibit significant therapeutic effects in treating inflammatory diseases such as middle ear inflammation (OM).

\section{Discussion}

In the present study, we showed that inhibition of PDE4B markedly enhanced upregulation of CYLD expression by the bacterial pathogen NTHi, thereby suggesting that PDE4B acts as a negative regulator for CYLD. Interestingly, inhibition of PDE4B a

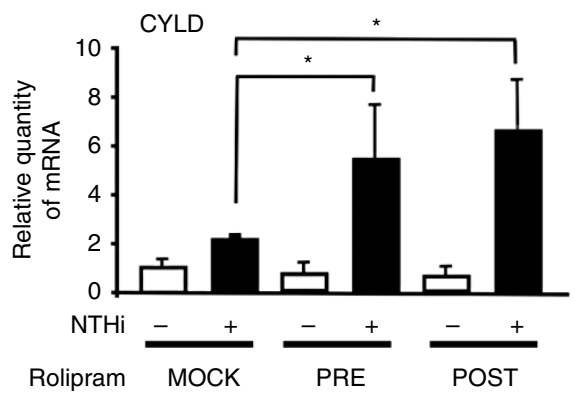

b

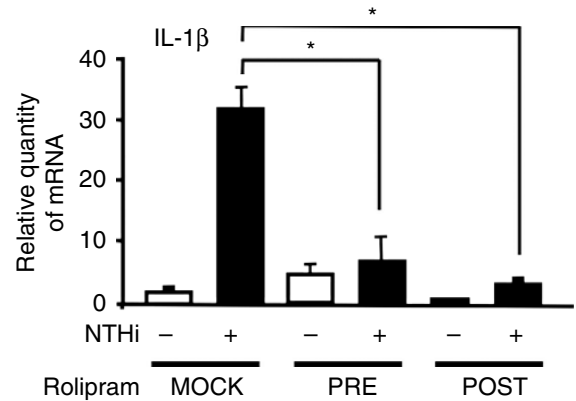

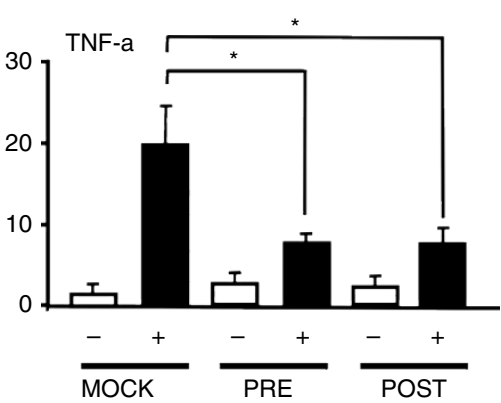

d Thickness
C

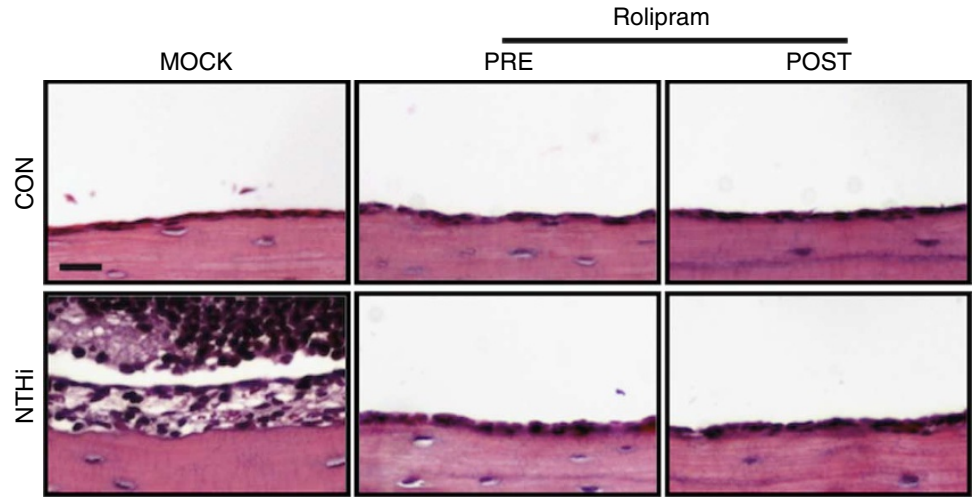

e

Rolipram

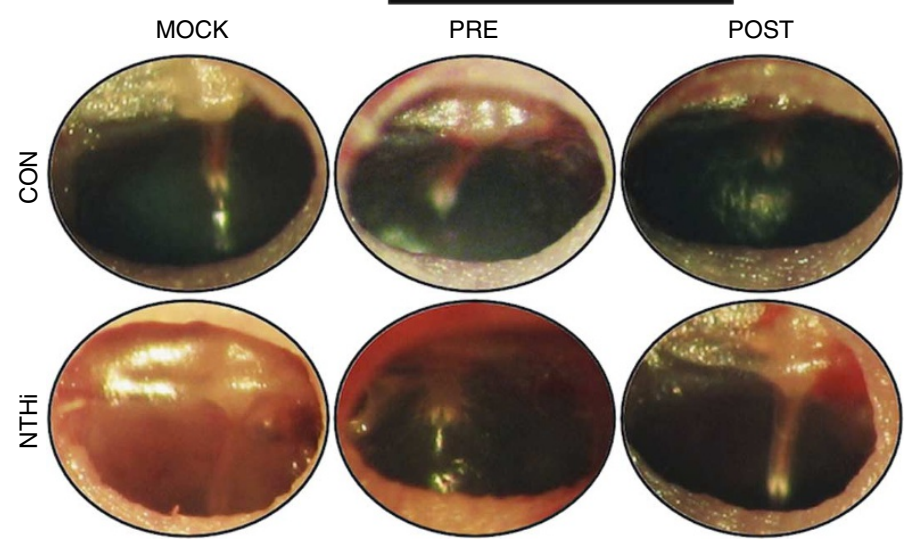

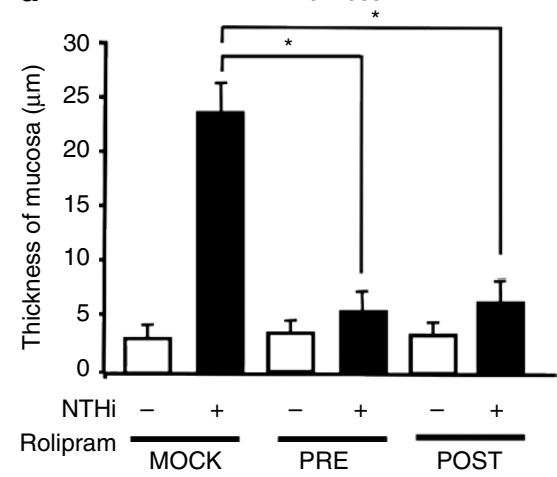

Figure 7 | Administration of specific PDE4 inhibitors upregulates CYLD and suppresses inflammation in a mouse otitis media model. mRNA expression of (a) CYLD and (b) pro-inflammatory cytokines was measured in middle ear tissues from C57BL/6J mice ototopically pre- or post-inoculated with Rolipram ( $50 \mu \mathrm{g}$ per ear) $3 \mathrm{~h}$ before (pretreatment) or $3 \mathrm{~h}$ after (post treatment) NTHi inoculation, and inoculated with NTHi for $9 \mathrm{~h}$. (c) Haematoxylin and eosin staining of middle ear tissues from $\mathrm{C} 57 \mathrm{BL} / 6 \mathrm{~J}$ mice ototopically pre- or post-inoculated with Rolipram ( $50 \mu \mathrm{g}$ per ear) $3 \mathrm{~h}$ before (pretreatment) or $3 \mathrm{~h}$ after (post treatment) NTHi inoculation, and inoculated with NTHi for $9 \mathrm{~h}$ (magnification $\times 400$ ). Scale bar, $20 \mu \mathrm{m}$. (d) Thickness of middle ear mucosa in C57BL/6J mice ototopically pre- or post-inoculated with Rolipram and inoculated with NTHi was measured from 15 middle ear tissue sections per experimental group. (e) Mice were transtympanically inoculated with NTHi with or without Rolipram ototopically pre- or post-treatment, and tympanic cavity was observed and recorded under the otoscope. Data in $\mathbf{a}, \mathbf{b}$ and $\mathbf{d}$ are mean \pm s.d. ( $n=3$ in $\mathbf{a}$ and $\mathbf{b}$, and 15 in $\mathbf{d})$. ${ }^{\star} P<0.05$. Statistical analysis was performed using Student's t-test. Data are representative of three or more independent experiments. CON, control; POST, post-inoculation; PRE, preinoculation. 
no longer suppressed NTHi-induced inflammation in Cylddeficient cells and mice. Moreover, PDE4B negatively regulates CYLD via selective activation of JNK2 but not JNK1. Importantly, ototopical post-inoculation administration of specific inhibitors for PDE4 suppressed NTHi-induced inflammation in a wellestablished mouse model of middle ear inflammation, thus demonstrating their therapeutic potential. Our studies offer novel insights into how inflammation is tightly regulated via inhibition of the expression level of CYLD, a key negative regulator for inflammation and may also lead to the development of new anti-inflammatory therapeutics by increasing the expression level of CYLD.

Of particular translational and therapeutic significance is that upregulating the expression level of CYLD, a key negative regulator of inflammation, via inhibition of its own negative regulator, $\mathrm{PDE} 4 \mathrm{~B}$, resulted in the suppression of bacteria-induced inflammation in well-established mouse models of lung and middle ear inflammation. In reviewing the past therapeutic strategies in developing anti-inflammatory agents, significant efforts have been focused on suppressing inflammation by directly targeting the positive signalling pathways ${ }^{7}$. For instance, suppressing IKK $\beta$ using small molecule inhibitors has been one of the focuses ${ }^{39}$. Although these agents exhibited certain efficacy, they, however, often resulted in significant adverse effects, for example, suppression of immune response, impairment of host defence and induction of apoptosis, which prevented their further clinical use in particular for long-term treatment ${ }^{8,9}$. Thus, there is an urgent need for developing novel therapeutic strategies without causing serious side effects, by targeting the negative instead of positive regulators of inflammation. Among all known negative regulators of inflammation, deubiquitinase CYLD has been shown to act as a key inducible negative feedback regulator for tightly controlling bacteria-induced inflammation, and thus preventing an overactive response in inflammatory diseases such as COPD and $\mathrm{OM}^{10,11}$. CYLD suppresses NF- $\mathrm{KB}$-dependent inflammation by removing K63-linked polyubiquitin chains from TRAF2, TRAF6, NEMO, TAK1, Bcl3 and RIP1 (ref. 13). In addition to regulating NF- $\kappa \mathrm{B}$-dependent inflammation, CYLD has also been implicated in a number of other cellular responses by targeting MAPK, JNK and p38, Akt, TCR signalling, cell cycle, calcium signalling and antiviral signalling pathways ${ }^{12,13}$. Mutations of CYLD have been reported to lead to the development of tumours such as CYLD ${ }^{14,15}$. In addition to mutations, dysregulated expression of CYLD has also been reported under various pathological conditions. We previously found that the expression of CYLD is relatively low under physiological conditions but is markedly upregulated by inflammatory insults such as $\mathrm{NTHi}^{16-}$ 18. In contrast, low expression of CYLD has also been reported in tumours $^{15,19}$. Interestingly, Cyld knockout mice exhibited no overt abnormalities and a normal lifespan ${ }^{20}$. Thus, it is plausible that increasing the expression level of CYLD by pharmacological inhibition of its own negative regulator may represent a novel and advantageous strategy for treating an overactive response in chronic inflammatory diseases without causing serious adverse effects often seen with targeting a positive regulator of inflammation. Indeed, in our present study, we provided direct in vitro and in vivo evidence for suppressing inflammation by upregulating the expression level of CYLD via specific inhibition of its own negative regulator PDE4B. It should be noted that inhibition of PDE4B via post-inoculation administration of PDE4 inhibitor also exhibited significant therapeutic effects on suppressing bacteria-induced inflammation in a well-established mouse model of middle ear inflammation. Our studies may provide novel therapeutic strategies for suppressing inflammation via directly upregulating its the negative regulators instead of inhibiting the positive regulators, thereby avoiding the serious side effects often seen when directly inhibiting positive pathway such as IKK $\beta$. In addition to treating inflammatory diseases, upregulating the expression level of CYLD may also lead to promising therapeutic strategies for tumours and fibrosis as the low expression of CYLD has been reported to have an important role in the development of tumours and lung fibrosis in patients.

Another major interesting finding in our studies is the identification of PDE4B as a critical negative regulator for upregulation of CYLD expression induced by bacteria. PDEs act as important regulators that both positively and negatively regulate cellular response via controlling the intracellular levels of second messengers cAMP and cGMP ${ }^{23-26}$. PDEs have long been thought as attractive and excellent therapeutic targets because of their unique tissue distribution, structural and functional properties, as well as sensitivity to selective inhibitors $^{21,22}$. Indeed, a number of PDE inhibitors have been already successfully developed as drugs in clinic, for example, Viagra (targeting PDE5) for erectile dysfunction and Roflumilast (targeting PDE4) for asthma and $\mathrm{COPD}^{21,27}$. However, the available general PDE4 inhibitors exhibited serious, sometimes intolerable, adverse effects due to its inhibitory effect on PDE4D ${ }^{29}$. The major side effects caused by inhibition of PDE4D include severe nausea, emesis and diarrhoea $21,28,29$. Moreover, inhibition of PDE4D also caused impaired growth ${ }^{30}$. Unfortunately, Roflumilast, the currently available drug, inhibits all of the four isotypes, A-D. Interestingly, our present study provides clear evidence that PDE4B acts as a key negative regulator for CYLD expression in vitro and in vivo. Inhibition of PDE4B markedly increased the expression of CYLD and subsequently suppressed bacteria-induced inflammation in wellestablished models of both lung and middle ear inflammation. Our studies may lead to the development of novel and more tolerable anti-inflammatory agent by selectively and specifically targeting PDE4B but not PDE4D for treating inflammatory disorders such as OM, which is extremely critical for children. In addition, our encouraging findings also showed that ototopical post-inoculation administration of PDE4 inhibitors significantly suppressed bacteria-induced inflammation. Thus, topical application of currently available drug Roflumilast may allow immediate and broad clinical application to treat inflammatory diseases to minimize the side effects caused due to systemic administration.

Of additional biological significance is our finding showing that PDE4B acts as a negative regulator for CYLD expression via selective activation of JNK2 but not JNK1. JNK family of MAPK has been shown to have a critical role in regulating a variety of both physiological and pathological responses ${ }^{33-35,40}$. JNK1 and JNK2 represent the major subfamily members of JNK. A number of studies indicate the functional differences and redundancy of JNK1 and JNK2 ${ }^{40-44}$. However, how JNK1 and 2 are selectively activated still remains largely unknown. In the present study, we provide direct evidence for the first time that PDE4B specifically and selectively mediates NTHi-induced activation of JNK2 but not JNK1 via a cAMP-PKA-dependent manner, which in turn leads to the negative regulation of CYLD expression. Thus, these studies may bring new insights into the upstream signalling mechanisms underlying specific and selective activation of JNK in inflammatory and infectious diseases.

\section{Methods}

Reagents and antibodies. Rolipram, 8-Br-cAMP, forskolin and cyclic nucleotide PDE assay kit were purchased from Enzo Life Sciences. PKI was purchased from EMD Millipore. SP600125 was purchased from A.G. Scientific. Roflumilast was purchased from Santa Cruz Biotechnology. Antibodies against phospho-stressactivated protein kinase/JNK (Thr-183/Tyr-185) and stress-activated protein 
kinase/JNK were purchased from Cell Signaling Technology. Antibody against CYLD was purchased from IMGENEX Corp. Antibodies against PDE4B and $\beta$-actin were purchased from Santa Cruz Biotechnology.

Bacterial strains and culture condition. Clinical isolates of NTHi strain 12 and three other NTHi strains were used in this study ${ }^{45,46}$. Bacteria were grown on chocolate agar plate at $37^{\circ} \mathrm{C}$ in an atmosphere of $5 \% \mathrm{CO}_{2}$ overnight and inoculated in brain heart infusion broth supplemented with $3.5 \mathrm{\mu g} \mathrm{ml}^{-1} \mathrm{NAD}$ and hemin. After overnight incubation, bacteria were subcultured into $5 \mathrm{ml}$ of fresh BHI and the log phase NTHi, monitored by measurement of optical density value, was washed and suspended in phosphate-buffered saline (PBS) for in vitro cell experiments and in isotonic saline for in vivo animal experiments.

Cell culture. All media described below were supplemented with $10 \%$ fetal bovine serum (Sigma-Aldrich) and Pen/Strep (100 units per ml penicillin and $0.1 \mathrm{mg}$ per $\mathrm{ml}$ streptomycin; Gibco). Human middle ear epithelial HMEEC cells were maintained in DMEM (Cellgro) supplemented with BEGM SingleQuots (Lonza). Human airway epithelial A549 cells were maintained in F-12K medium (Gibco) as described previously ${ }^{45,46}$. Human primary bronchial epithelial NHBE (Lonza) cells were maintained in bronchial epithelial growth media (BEGM) supplemented with BEGM SingleQuots ${ }^{12}$. Air-liquid culture of human primary NHBE cells was conducted as described previously ${ }^{47}$. Wild-type (WT) and Cyld ${ }^{-/-}$MEF cells were obtained from E13 embryos and maintained in DMEM. WT and Cyld ${ }^{-1}$ MEFs and mice were obtained as described previously ${ }^{12,20}$. All cells were cultured in a humidified atmosphere of $5 \% \mathrm{CO}_{2}$ at $37^{\circ} \mathrm{C}$.

Real-time quantitative RT-PCR analysis. Total RNA was isolated with TRIzol reagent (Invitrogen) by following the manufacturer's instruction. For the reverse transcription reaction, TaqMan reverse transcription reagents (Applied Biosystems) were used as described ${ }^{18,47}$. PCR amplifications were performed by using SYBR Green Universal Master Mix (Applied Biosystems). In brief, reactions were performed in triplicate containing $2 \times$ universal master mix, $1 \mu \mathrm{l}$ of template cDNA, $500 \mathrm{nM}$ primers in a final volume of $12.5 \mu \mathrm{l}$, and they were analysed in a 96-well optical reaction plate (Applied Biosystems). Reactions were amplified and quantified by using an ABI 7500 sequence detector and the manufacturer's corresponding software (7000v1.3.1; Applied Biosystems). The relative quantities of mRNAs were obtained by using the comparative $\mathrm{Ct}$ method and were normalized using human cyclophilin or mouse glyceraldehydes-3-phosphate dehydrogenase as an endogenous control. The primers for human IL-1 $\beta$, IL- 8 , TNF- $\alpha$, CYLD, PDE4A, PDE4B, JNK1, JNK2 and cyclophilin, as well as mouse IL-1 $\beta$, MIP-2, TNF- $\alpha$, CYLD, PDE4A, PDE4B and glyceraldehydes-3-phosphate dehydrogenase were described previously $16,32,35,48$. Human PDE4C: $5^{\prime}$-GACTTACCCCTC GACAACCA- $3^{\prime}$ and $5^{\prime}$-GAAAGTCTGCCTGCCAAGAG-3'; human PDE4D: $5^{\prime}$-TGCCATCTGTTGATCAGGAA- $3^{\prime}$ and $5^{\prime}$-CCCAGTTGTGTTTCCGAGTT- ${ }^{\prime}$; mouse PDE4C: $5^{\prime}$-CATGCAGTCGGACTCTGTGT- $3^{\prime}$ and $5^{\prime}$-GGACTGGTGAAG GCAACATT-3'; mouse PDE4D: 5'-CTCTGTGCACCCAAGACTCA-3' and 5'-CAAGTTTCAGGCTGGCTTTC-3'.

Plasmids, transfections and luciferase assay. The expression plasmids p65 WT, a constitutively active form of IKK $\beta$ (IKK $\beta$-CA), and the luciferase reporter construct of NF- $\kappa B$ were previously described ${ }^{49,50}$. Empty vector was used as a control and was also added where necessary to ensure an equivalent amount of input DNA. All transient transfections were carried out in triplicate using TransIT-LT1 reagent (Mirus) following the manufacturer's instruction. For experiments with inhibitors, the transfected cells were pretreated with or without chemical inhibitors for $1 \mathrm{~h}$ followed by 5 -h incubation with NTHi lysates. Luciferase activity was normalized with respect to $\beta$-galactosidase activity. Data from all experiments are presented as the relative luciferase activity (mean \pm s.d.) from at least three independent sets of experiments, each with triplicate measurements.

RNA-mediated interference. RNA-mediated interference for downregulating CYLD expression was carried out using PSUPER-CYLD as described previously ${ }^{16,18}$ and the sequence for the siCYLD is $5^{\prime}$-GATCCCCGAGCTACTGAGG ACAGAAATTCAAGAGATTTCTGTCCTCAGTAGCTCTTTTTGGAAA- ${ }^{\prime}$. Human siRNAs for PDE4B, JNK1 and JNK2 were purchased from Dharmacon, and knockdown of PDE4B, JNK1 and JNK2, using siPDE4B, siJNK1 and siJNK2, was performed using Lipofectamine 2000 (Invitrogen) following the manufacturer's instruction. ON-TARGETplus SMARTpool of siRNAs targeting human PDE4B, human JNK1 and human JNK2 consists of four siRNAs and sequences for the siRNAs are as follows: Human siPDE4B ( $5^{\prime}$-AGUUACAAGUUC AGGCGUU- $3^{\prime}, 5^{\prime}$-GCUCAGGACAUUCUCGAUA-3', $5^{\prime}$-AGACAAUGGUAGAA ACGAA- $3^{\prime}, 5^{\prime}$-GCAUUCAGGUCCUUCGCAA- $3^{\prime}$ ); human siJNK1 (5'-GAUGAC GCCUUAUGUAGUG-3', 5'-GAAUAGUAUGCGCAGCUUA-3', 5'-GGCAUGG GCUACAAGGAAA- $3^{\prime}, 5^{\prime}$-GCCCAGUAAUAUAGUAGUA-3'); human siJNK2 (5'-GAUUGUUUGUGCUGCAUUU-3', 5'-GGCUGUCGAUGAUAGGUUA-3', $5^{\prime}$-AGCCAACUGUGAGGAAUUA-3' ${ }^{\prime}$ ' ${ }^{\prime}$-UCGUGAACUUGUCCUCUUA-3').
Western blot analysis. Western blot analysis was performed as described previously and following the manufacturer's instruction ${ }^{18}$. Western blots were performed using whole-cell extracts, separated on 6 or $8 \%$ SDS-polyacrylamide gel electrophoresis gels, and transferred to polyvinylidene difluoride membranes. The membrane was blocked with a solution of Tris-buffered saline (TBS) containing $0.1 \%$ Tween 20 (TBS-T) and 5\% non-fat dry milk. The membrane was then incubated in a 1:2,000 dilution of a primary antibody in 5\% BSA-TBS-T. After three washes in TBS-T, the membrane was incubated with 1:5,000 dilution of the corresponding secondary antibody in $5 \%$ non-fat dry milk-TBS-T. Respective proteins were visualized by using Amersham ECL Prime Regent (GE Healthcare Biosciences).

Immunoprecipitation and PDE assay. To conduct the selective immunoprecipitation of PDE4B, cell lysates were incubated with $1 \mu \mathrm{g}$ of primary antibodies overnight at $4{ }^{\circ} \mathrm{C}$, followed by 2 -h incubation with protein $\mathrm{A} / \mathrm{G}$-agarose beads (Santa Cruz Biotechnology). Immunoprecipitates were washed three times with $\mathrm{PBS}$ and one time with PDE assay buffer $(10 \mathrm{mM}$ Tris- $\mathrm{HCl}, \mathrm{pH}$ 7.4), and then used for PDE assay. PDE activity was measured by using cyclic nucleotide PDE assay kit (Enzo Life Sciences) following the instructions. PDE activity of PDE4B immunoprecipitates was blocked in the presence of $10 \mu \mathrm{M}$ Rolipram.

Mice and animal experiments. $C y l d^{-/-}$mice have been described previously ${ }^{12,20}$, and age-matched background C57BL/6J mice were used as WT controls. For NTHi-induced inflammation in WT and Cyld ${ }^{-/-}$mice, anaesthetized mice were transtympanically or intratracheally inoculated with NTHi at a concentration of $1 \times 10^{7}$ or $5 \times 10^{7}$ c.f.u. per mouse, respectively, and saline was inoculated as control. The inoculated mice were then killed $9 \mathrm{~h}$ after NTHi inoculation. Eardrums of mice were inspected for signs of middle ear inflammation and photographed for recording pathological changes of eardrum under the otoscope. Dissected temporal bones and lungs were then subjected to total RNA extraction and histological analysis. For polymorphonuclear neutrophil analysis, bronchoalveolar lavage was performed by cannulating the trachea with sterilized PBS in WT and Cyld ${ }^{-/-}$mice $^{17}$. For inhibition study, mice were pretreated with chemical inhibitors i.p. $2 \mathrm{~h}$ before NTHi inoculation. For the ototopical pretreatment experiments, mice were pretreated with Rolipram through tympanic membrane $3 \mathrm{~h}$ before NTHi inoculation, and inoculated with NTHi for $9 \mathrm{~h}$. For the ototopical post-treatment experiments, mice were inoculated with NTHi first, and ototopically post-treated with Rolipram through tympanic membrane $3 \mathrm{~h}$ after NTHi inoculation. The inoculated mice were killed $9 \mathrm{~h}$ after NTHi inoculation. All animal experiments were approved by the Institutional Animal Care and Use Committee at Georgia State University.

Histology and IF assay. For histological analysis, formalin-fixed paraffinembedded lung and middle ear tissues were sectioned $(4 \mu \mathrm{m})$, and then stained with haematoxylin and eosin to visualize inflammatory responses and pathological changes in the middle ear or lung. IF detection of CYLD and PDE4B proteins were performed using rabbit anti-CYLD, rabbit anti-PDE4B and FITC-conjugated goat anti-rabbit IgG (Santa Cruz Biotechnology) in the paraffin section of mouse middle ear or lung tissue. Stained sections were then imaged, and images were recorded under light and fluorescence microscopy systems (AxioVert $40 \mathrm{CFL}$, AxioCam MRC and AxioVision LE Image system, Carl Zeiss).

Measurement of mucosal thickness in the middle ear. Mucosal thickness in the middle ear cavity was measured from 15 sections per each experimental group using AxioVision LE Image system software (Carl Zeiss) to measure and quantify the degree of inflammation such as mucosal hyperplasia and inflammatory cell infiltration. The average of mucosal thickness from 15 sections was presented in a bar graph as the mean \pm s.d. along with representative tissue sections.

Statistical analysis. All experiments were repeated at least three times with consistent results. Data are shown as mean \pm s.d. Statistical analysis was assessed by two-tailed unpaired student's $t$-test. $P<0.05$ was considered statistically significant.

\section{References}

1. Grivennikov, S. I., Greten, F. R. \& Karin, M. Immunity, inflammation, and cancer. Cell 140, 883-899 (2010).

2. Decramer, M., Janssens, W. \& Miravitlles, M. Chronic obstructive pulmonary disease. Lancet 379, 1341-1351 (2012).

3. Leichtle, A., Lai, Y., Wollenberg, B., Wasserman, S. I. \& Ryan, A. F. Innate signaling in otitis media: pathogenesis and recovery. Curr. Allergy Asthma Rep. 11, 78-84 (2011)

4. Nathan, C. \& Ding, A. Nonresolving inflammation. Cell 140, 871-882 (2010)

5. Medzhitov, R. Origin and physiological roles of inflammation. Nature 454, 428-435 (2008).

6. Liew, F. Y., Xu, D., Brint, E. K. \& O’Neill, L. A. Negative regulation of toll-like receptor-mediated immune responses. Nat. Rev. Immunol. 5, 446-458 (2005). 
7. Liu, F., Xia, Y., Parker, A. S. \& Verma, I. M. IKK biology. Immunol. Rev. 246, 239-253 (2012).

8. Niederberger, E. \& Geisslinger, G. The IKK-NF-kappaB pathway: a source for novel molecular drug targets in pain therapy? FASEB J. 22, 3432-3442 (2008).

9. Chen, L. W. et al. The two faces of IKK and NF-kappaB inhibition: prevention of systemic inflammation but increased local injury following intestinal ischemia-reperfusion. Nat. Med. 9, 575-581 (2003).

10. Sun, S. C. Deubiquitylation and regulation of the immune response. Nat. Rev. Immunol. 8, 501-511 (2008).

11. Wang, W. Y., Lim, J. H. \& Li, J. D. Synergistic and feedback signaling mechanisms in the regulation of inflammation in respiratory infections. Cell. Mol. Immunol. 9, 131-135 (2012).

12. Lim, J. H. et al. CYLD negatively regulates transforming growth factor-betasignalling via deubiquitinating Akt. Nat. Commun. 3, 771 (2012).

13. Sun, S. C. CYLD: a tumor suppressor deubiquitinase regulating NF-kappaB activation and diverse biological processes. Cell Death Differ. 17, 25-34 (2010).

14. Bignell, G. R. et al. Identification of the familial cylindromatosis tumoursuppressor gene. Nat. Genet. 25, 160-165 (2000).

15. Massoumi, R. CYLD: a deubiquitination enzyme with multiple roles in cancer. Future Oncol. 7, 285-297 (2011).

16. Jono, H. et al. NF-kappaB is essential for induction of CYLD, the negative regulator of NF-kappaB: evidence for a novel inducible autoregulatory feedback pathway. J. Biol. Chem. 279, 36171-36174 (2004).

17. Lim, J. H. et al. Tumor suppressor CYLD acts as a negative regulator for nontypeable Haemophilus influenza-induced inflammation in the middle ear and lung of mice. PLoS One 2, e1032 (2007).

18. Yoshida, H., Jono, H., Kai, H. \& Li, J. D. The tumor suppressor cylindromatosis (CYLD) acts as a negative regulator for toll-like receptor 2 signaling via negative cross-talk with TRAF6 AND TRAF7. J. Biol. Chem. 280, 41111-41121 (2005).

19. Espinosa, L. et al. The Notch/Hes1 pathway sustains NF-kappaB activation through CYLD repression in T cell leukemia. Cancer Cell 18, 268-281 (2010).

20. Lim, J. H. et al. Tumor suppressor CYLD regulates acute lung injury in lethal Streptococcus pneumoniae infections. Immunity 27, 349-360 (2007).

21. Michalski, J. M., Golden, G., Ikari, J. \& Rennard, S. I. PDE4: a novel target in the treatment of chronic obstructive pulmonary disease. Clin. Pharmacol. Ther. 91, 134-142 (2012).

22. Bender, A. T. \& Beavo, J. A. Cyclic nucleotide phosphodiesterases: molecular regulation to clinical use. Pharmacol. Rev. 58, 488-520 (2006).

23. Dyachok, O., Isakov, Y., Sagetorp, J. \& Tengholm, A. Oscillations of cyclic AMP in hormone-stimulated insulin-secreting beta-cells. Nature 439, 349-352 (2006).

24. Fischmeister, R. et al. Compartmentation of cyclic nucleotide signaling in the heart: the role of cyclic nucleotide phosphodiesterases. Circ. Res. 99, 816-828 (2006)

25. Nikolaev, V. O., Gambaryan, S., Engelhardt, S., Walter, U. \& Lohse, M. J. Realtime monitoring of the PDE2 activity of live cells: hormone-stimulated cAMP hydrolysis is faster than hormone-stimulated cAMP synthesis. J. Biol. Chem. 280, 1716-1719 (2005).

26. Nikolaev, V. O., Gambaryan, S. \& Lohse, M. J. Fluorescent sensors for rapid monitoring of intracellular cGMP. Nat. Methods 3, 23-25 (2006).

27. Page, C. P. \& Spina, D. Selective PDE inhibitors as novel treatments for respiratory diseases. Curr. Opin. Pharmacol. 12, 275-286 (2012).

28. Lipworth, B. J. Phosphodiesterase-4 inhibitors for asthma and chronic obstructive pulmonary disease. Lancet 365, 167-175 (2005).

29. Banner, K. H. \& Press, N. J. Dual PDE3/4 inhibitors as therapeutic agents for chronic obstructive pulmonary disease. Br. J. Pharmacol. 157, 892-906 (2009).

30. Jin, S. L., Richard, F. J., Kuo, W. P., D’Ercole, A. J. \& Conti, M. Impaired growth and fertility of cAMP-specific phosphodiesterase PDE4D-deficient mice. Proc. Natl Acad. Sci. USA 96, 11998-12003 (1999).

31. Barber, R. et al. Differential expression of PDE4 cAMP phosphodiesterase isoforms in inflammatory cells of smokers with COPD, smokers without COPD, and nonsmokers. Am. J. Physiol. Lung Cell Mol. Physiol. 287, L332-L343 (2004)

32. Lee, J. et al. Phosphodiesterase 4B mediates extracellular signal-regulated kinase-dependent up-regulation of mucin MUC5AC protein by Streptococcus pneumoniae by inhibiting cAMP-protein kinase A-dependent MKP-1 phosphatase pathway. J. Biol. Chem. 287, 22799-22811 (2012).

33. Wang, X. \& Liu, Y. Regulation of innate immune response by MAP kinase phosphatase-1. Cell Signal. 19, 1372-1382 (2007).

34. Amdekar, S., Singh, V. \& Singh, D. D. Probiotic therapy: immunomodulating approach toward urinary tract infection. Curr. Microbiol. 63, 484-490 (2011).

35. Ha, U. H. et al. MKP1 regulates the induction of MUC5AC mucin by Streptococcus pneumoniae pneumolysin by inhibiting the PAK4-JNK signaling pathway. J. Biol. Chem. 283, 30624-30631 (2008).
36. Herr, B. D. \& Marzo, S. J. Intratympanic steroid perfusion for refractory sudden sensorineural hearing loss. Otolaryngol. Head Neck Surg. 132, 527-531 (2005).

37. Rahman, A., Rizwan, S., Waycaster, C. \& Wall, G. M. Pooled analysis of two clinical trials comparing the clinical outcomes of topical ciprofloxacin/ dexamethasone otic suspension and polymyxin $\mathrm{B} /$ neomycin/hydrocortisone otic suspension for the treatment of acute otitis externa in adults and children. Clin. Ther. 29, 1950-1956 (2007).

38. Stenner, M., Jecker, P., Gouveris, H. \& Mann, W. Treatment of sensorineural hearing loss in acute viral otitis media with intratympanic dexamethasone and hyaluronic acid in comparison with intravenous therapy. Laryngorhinootologie 85, 32-37 (2006)

39. Barnes, P. J. The cytokine network in asthma and chronic obstructive pulmonary disease. J. Clin. Invest. 118, 3546-3556 (2008).

40. Bode, A. M. \& Dong, Z. The functional contrariety of JNK. Mol. Carcinog. 46, 591-598 (2007).

41. Sabapathy, K. et al. Distinct roles for JNK1 and JNK2 in regulating JNK activity and c-Jun-dependent cell proliferation. Mol. Cell 15, 713-725 (2004).

42. Blonska, M. et al. The CARMA1-Bcl10 signaling complex selectively regulates JNK2 kinase in the T cell receptor-signaling pathway. Immunity 26, 55-66 (2007).

43. Liu, J., Minemoto, Y. \& Lin, A. c-Jun N-terminal protein kinase 1 (JNK1), but not JNK2, is essential for tumor necrosis factor alpha-induced c-Jun kinase activation and apoptosis. Mol. Cell Biol. 24, 10844-10856 (2004).

44. Lu, Z. et al. Disruption of JNK2 decreases the cytokine response to Plasmodium falciparum glycosylphosphatidylinositol in vitro and confers protection in a cerebral malaria model. J. Immunol. 177, 6344-6352 (2006).

45. Imasato, A. et al. Inhibition of p38 MAPK by glucocorticoids via induction of MAPK phosphatase-1 enhances nontypeable Haemophilus influenzae-induced expression of toll-like receptor 2. J. Biol. Chem. 277, 47444-47450 (2002).

46. Wang, B. et al. Novel cytoplasmic proteins of nontypeable Haemophilus influenzae up-regulate human MUC5AC mucin transcription via a positive p38 mitogen-activated protein kinase pathway and a negative phosphoinositide 3-kinase-Akt pathway. J. Biol. Chem. 277, 949-957 (2002).

47. Sakai, A. et al. The bacterium, nontypeable Haemophilus influenzae, enhances host antiviral response by inducing Toll-like receptor 7 expression: evidence for negative regulation of host anti-viral response by CYLD. FEBS J. 274, 3655-3668 (2007).

48. Jeon, K. I. et al. Vinpocetine inhibits NF-kappaB-dependent inflammation via an IKK-dependent but PDE-independent mechanism. Proc. Natl Acad. Sci. USA 107, 9795-9800 (2010).

49. Ishinaga, H. et al. TGF-beta induces p65 acetylation to enhance bacteriainduced NF-kappaB activation. EMBO J. 26, 1150-1162 (2007).

50. Xu, X. et al. EVI1 Acts as an inducible negative-feedback regulator of NF-kappaB by inhibiting p65 acetylation. J. Immunol. 188, 6371-6380 (2012).

\section{Acknowledgements}

We thank Wenzhuo Y. Wang for her assistance with editing this manuscript. J.-D.L. is Georgia Research Alliance Eminent Scholar. This work was supported by grants from National Institutes of Health DC005843 and DC004562 to J.-D.L.

\section{Author contributions}

K.K. and J.Y.L. conceived, designed and performed experiments. M.M., J.H.L., H.J. and T.K. carried out experiments and analysed data. H.X., C.Y. and H.K. contributed reagents and materials, and performed data analysis. J.-D.L. was responsible for overall study design and data analysis, and he wrote the manuscript and supervised the project.

\section{Additional information}

Supplementary Information accompanies this paper at http://www.nature.com/ naturecommunications

Competing financial interests: The authors declare no competing financial interests.

Reprints and permission information is available online at http://npg.nature.com/ reprintsandpermissions/

How to cite this article: Komatsu, K. et al. Inhibition of PDE4B suppresses inflammation by increasing expression of the deubiquitinase CYLD. Nat. Commun. 4:1684 doi: $10.1038 /$ ncomms2674 (2012)

(c) $(\$$ This work is licensed under a Creative Commons Attributioncc. this license, visit http://creativecommons.org/licenses/by-nc-nd/3.0/ 